\title{
The Effect of Grain Size Distribution on Nonlinear Flow Behavior in Sandy Porous Media
}

\author{
Jan H. van Lopik ${ }^{1}$ - Roy Snoeijers ${ }^{1}$ - Teun C. G. W. van Dooren ${ }^{1}$ • \\ Amir Raoof ${ }^{1}$. Ruud J. Schotting ${ }^{1}$
}

Received: 7 March 2017 / Accepted: 19 July 2017 / Published online: 24 August 2017

(C) The Author(s) 2017. This article is an open access publication

\begin{abstract}
The current study provides new experimental data on nonlinear flow behavior in various uniformly graded granular materials (20 samples) ranging from medium sands $\left(d_{50}>0.39 \mathrm{~mm}\right)$ to gravel $\left(d_{50}=6.3 \mathrm{~mm}\right)$. Generally, theoretical equations relate the Forchheimer parameters $a$ and $b$ to the porosity, as well as the characteristic pore length, which is assumed to be the median grain size $\left(d_{50}\right)$ of the porous medium. However, numerical and experimental studies show that flow resistance in porous media is largely determined by the geometry of the pore structure. In this study, the effect of the grain size distribution was analyzed using subangular-subrounded sands and approximately equal compaction grades. We have used a reference dataset of 11 uniformly graded filter sands. Mixtures of filter sands were used to obtain a slightly more well-graded composite sand (increased $C_{u}$ values by a factor of 1.19 up to 2.32) with respect to its associated reference sand at equal median grain size $\left(d_{50}\right)$ and porosity. For all composite sands, the observed flow resistance was higher than in the corresponding reference sand at equal $d_{50}$, resulting in increased $a$ coefficients by factors up to 1.68 , as well as increased $b$ coefficients by factors up to 1.44 . A modified Ergun relationship with Ergun constants of 139.1 for $A$ and 2.2 for $B$, as well as the use of $d_{m}-\sigma$ as characteristic pore length predicted the coefficients $a$ and $b$ accurately.
\end{abstract}

Keywords Forchheimer equation · Nonlinear flow · Filter sand · Grain size distribution · Column experiment

\section{Introduction}

The understanding of nonlinear liquid or gas flow behavior in natural and artificial porous media, such as rock, consolidated or unconsolidated material and packed-bed-reactors is a key factor in reservoir engineering applications (Wen et al. 2011; Mijic et al. 2013; Yeh and

Jan H. van Lopik

j.h.vanlopik@uu.nl

1 Department of Earth Sciences, Environmental Hydrogeology group, Utrecht University,

Princetonplein 9, 3584 CC Utrecht, The Netherlands 
Chang 2013; Houben 2015) and chemical and technical industries (e.g., Jafari et al. 2008). In the field of reservoir engineering, fluid flow behavior near the well screen controls the efficiency of oil/gas production wells (e.g., Holditz and Morse 1976; Aulisa et al. 2009), as well as the efficiency of injection/extraction wells in groundwater resources applications (e.g., Sen 1989; Mathias and Todman 2010; Wen et al. 2011, 2013; Yeh and Chang 2013; Houben 2015; Mathias and Moutsopoulos 2016). Moreover, nonlinear flow at full-aquifer scale in a broad range of geological formations, such as gravel, karstic or fractured aquifers, is widely addressed in the literature (e.g., Moutsopoulos and Tsihrintzis 2005; Moutsopoulos 2007).

Commonly, groundwater flow near wells is described using the classical Darcy's law (Darcy 1856), as:

$$
i=-\frac{1}{K} q
$$

where $i$ is the hydraulic gradient $(\mathrm{m} / \mathrm{m}), K$ is the hydraulic conductivity of the porous medium $(\mathrm{m} / \mathrm{s})$ and $q$ is the specific discharge $(\mathrm{m} / \mathrm{s})$. This empirical linear relationship for fluid flow in porous media is only applicable for a specific regime of flow where flow velocities are sufficiently low, and it becomes less accurate as flow velocities become larger. The deviation of the nonlinear relationship between specific discharge and hydraulic gradient due to increased flow resistance at higher flow velocities has been widely addressed in the literature (e.g., Forchheimer 1901; Izbash 1931; Sidiropoulou et al. 2007). Forchheimer (1901) described the nonlinear behavior adding a second-order quadratic term to the Darcy's law:

$$
i=-a q-b q^{2}
$$

Comparing Eqs. 1 and 2, $a(\mathrm{~s} / \mathrm{m})$ is a parameter equal to the reciprocal of the hydraulic conductivity (i.e., $a=1 / K)$ and $b$ is the empirical Forchheimer coefficient $\left(\mathrm{s}^{2} / \mathrm{m}^{2}\right)$. The Forchheimer equation is able to accurately fit the experimental data in packed-column experiments with granular material for most cases of non-Darcy flows (Sidiropoulou et al. 2007; Moutsopoulos et al. 2009; Sedghi-Asl et al. 2014; Li et al. 2017). Mathias and Moutsopoulos (2016) showed that the Forchheimer equation should be used instead of Darcy's law to account for additional head losses due to non-Darcy flow conditions in the vicinity of injection and production wells. Other analytical studies use the Forchheimer equation to predict non-steady-state, nonlinear flow in gravel aquifers at early time steps when hydraulic gradients are steep (e.g., Moutsopoulos and Tsihrintzis 2005; Moutsopoulos 2007).

Besides the Forchheimer equation, other empirical equations have been proposed to describe the relation between specific discharge and hydraulic gradient for nonlinear flow (Izbash 1931; Irmay 1958; Bear 1972). For example, the Izbash equation' [initially proposed by Forchheimer (1901)], which is a nonlinear power law function, is widely used to model the non-Darcy flow (e.g., Bordier and Zimmer 2000; Wen et al. 2013). Despite the experimental validity of the Izbash equation, it lacks any physical base (Bordier and Zimmer 2000).

Many experimental data on (post-Darcy) nonlinear flow in porous media are available for coarser porous media, such as coarse sands, gravels and glass beads (Sidiropoulou et al. 2007; Moutsopoulos et al. 2009; Sedghi-Asl and Rahimi 2011; Huang et al. 2013; Bağci et al. 2014; Salahi et al. (2015); Sedghi-Asl et al. 2014; Li et al. 2017). A relatively small amount of experimental data is available for finer unconsolidated sandy porous media. Moreover, most studies only provided porosity and the median $\left(d_{50}\right)$ or 
mean grain size to describe the porous media. Relationships to predict Forchheimer coefficients $a$ and $b$ are based on these two parameters (e.g., Ergun 1952; Macdonald et al. 1979; Kovács 1981; Kadlec and Knight 1996; Sidiropoulou et al. 2007). However, numerical simulations, as well as experimental studies, showed that the geometry of the pore structure have a large influence on flow behavior (e.g., Hill and Koch 2002; Li and Ma 2011; Allen et al. 2013; Koekemoer and Luckos 2015). Here, we investigated the effect of nonlinear flow behavior empirically for granular porous media (filter sand) with various grain size distributions for a given reference median grain size $\left(d_{50}\right)$ and compaction grade.

\section{Background}

\subsection{Criteria for Nonlinear Flow in Porous Media}

Experimental and numerical studies have investigated the upper limits of the Darcy regime for fluid flow in porous media. Many attempts have been done to define a criterion to describe the upper limit of the Darcy regime. Formerly, it was believed that nonlinear flow behavior in porous media was solely due to turbulent flow (inertial energy losses by highly chaotic and unsteady flow).

The Reynolds number is used to indicate whether fluid flow is laminar or turbulent. It is defined as (Bear 1972):

$$
R e=\frac{\rho d_{50} q}{\mu}=\frac{\text { inertial forces }}{\text { viscous forces }}
$$

where $\rho$ is the fluid density $\left(\mathrm{kg} / \mathrm{m}^{3}\right), d_{50}$ is the median grain size of the porous medium as a proxy for the characteristic pore length $(\mathrm{m}), q$ is the specific discharge $(\mathrm{m} / \mathrm{s})$, and $\mu$ is the dynamic viscosity $(\mathrm{kg} / \mathrm{m} \mathrm{s})$. Besides Reynolds number, which is solely based on the average particle size of the porous medium to define the properties of the medium, various other criterions have been proposed. For example, Ergun (1952) used a modified Reynolds number by including porosity, while Comiti et al. (2000) used a pore Reynolds number based on porosity, tortuosity and the dynamic specific surface area of the pore. Zhengwen and Grigg (2006) stated that determination of a representative pore diameter as characteristic length of the porous media is difficult, due to the complexity of pore structures. Moreover, flow through a complex pore structure significantly differs from flow in a conduit, and, hence, no sharp transition between laminar and turbulent flow can be observed in porous media. A wide range of critical particle Reynolds numbers is given in the literature for the onset of nonlinear laminar flow. For example, Bear (1972), Hassanizadeh and Gray (1987), Ma and Ruth (1993) and Comiti et al. (2000) suggested critical particle Reynolds numbers ranging between 1 and 15. Other studies, such as Scheidegger (1974), obtained higher maximal critical particle Reynolds numbers up to a value of 75 .

Ma and Ruth (1993) and Lage (1998) introduced new criteria to evaluate the onset of nonlinear flow by means of a "Forchheimer number," based on the intrinsic permeability and the non-Darcy coefficient $b$ in the Forchheimer equation. However, such criteria require experimentally or empirically determined values for the Forchheimer coefficients $a$ and $b$, while criteria such as the particle Reynolds number require only the average grain size in order to estimate the onset of nonlinear flow in a given porous media. 


\subsection{Nonlinear Laminar Flow and Fully Turbulent Flow in Porous Media}

Traditionally, it was believed that the onset of nonlinear flow was solely due to energy losses by the occurrence of turbulence (e.g., Ergun 1952; Ward 1964). However, flow visualization studies in porous media showed that turbulent flow occurs at much higher particle Reynold numbers than the critical particle Reynold numbers of 1-15 for the onset of nonlinear flow (e.g., Chauveteau and Thirriot 1967; Dybbs and Edwards 1984; Seguin et al. 1998). Critical particle Reynolds number of 300-533 was determined for the onset of the turbulent flow regime using a uniform spheres packing with particle diameters ranging from 0.5 to $8 \mathrm{~cm}$, respectively (Jolls and Hanratty 1966; Latifi et al. 1989; Rode et al. 1994; Seguin et al. 1998), as well as for cylindrical or plate arrangements (Dybbs and Edwards 1984). Hence, a nonlinear laminar flow regime should be considered before fully turbulent flow develops in porous medium. A smooth transition was observed from nonlinear laminar flow, to the onset of small vortices and eddies in some pore spaces, to eventually, development of fully turbulent flow in the porous medium (e.g., Dybbs and Edwards 1984; Seguin et al. 1998). In the literature, this nonlinearity in the laminar flow regime is ascribed to different microscale processes (e.g., Hassanizadeh and Gray 1987; Ma and Ruth 1993; Skjetne and Auriault 1999; Hill and Koch 2002; Nield 2002). In general, it is assumed that the deviation from the linear Darcy's law at increased Reynolds numbers is due to the occurrence of microscopic inertial forces (e.g., Bear 1972; Macdonald et al. 1979; Venkataraman and Rao 1998). Other studies, such as Panfilov et al. (2003) and Panfilov and Fourar (2006), showed that the main mechanisms of energy dissipation that contribute to the nonlinear flow term are due to both recirculation of eddies in closed streamline regions, as well as streamline deformation in the main area of flow. Ma and Ruth (1993) showed that the onset of nonlinearity cannot be fully described by microscopic inertial terms using a simple tube model. Hassanizadeh and Gray (1987) stated that the onset of nonlinear flow behavior is due to the occurrence of interfacial drag forces at increased flow velocities and inertial forces become significantly important at particle Reynold numbers of about 100 . The effect of the viscous boundary layer on drag was addressed by Whitaker (1996). These studies showed that microscopic analysis should be used to fully understand macroscopic nonlinear laminar flow behavior in a given complex pore structure for a given Reynolds number. Overall, the geometry of the pore structure (such as tube-like or blob-like pore surfaces), as well as the total fluid-solid interface and its interface roughness, largely determine the flow resistance due to drag force terms in the Forchheimer coefficient $b$ (e.g., Ma and Ruth 1993; Liu et al. 1995; Thauvin and Mohanty 1998; Panfilov and Fourar 2006; Chaudhary et al. 2013).

As described above, the macroscopic transition from laminar Darcy flow, to nonlinear laminar flow to eventually fully turbulent flow, is a gradual process (e.g., Seguin et al. 1998). Many experimental datasets on nonlinear flow behavior in granular porous media show that this gradual process can be accurately predicted by the Forchheimer equation (Eq. 2) for various ranges of particle Reynold numbers in the nonlinear laminar flow regime, as well as in the turbulent flow regime (e.g., Venkataraman and Rao 1998; Sidiropoulou et al. 2007; Moutsopoulos et al. 2009; Bağci et al. 2014; Sedghi-Asl et al. 2014; Salahi et al. 2015; Li et al. 2017).

For example, Li et al. (2017) conducted flow experiments on sands at Reynolds numbers below 40 and, hence, investigated the onset of the nonlinear laminar regime. By contrast, Sedghi-Asl et al. (2014) investigated the Forchheimer coefficients of sands and gravel experimentally at Reynold numbers ranging from 31 to 720 (for a sand with $d_{50}$ of $5.5 \mathrm{~mm}$ ) up to $2400-30,750$ (for a gravel with $d_{50}$ of $56.8 \mathrm{~mm}$ ). Moutsopoulos et al. (2009) evaluated coefficients $a$ and $b$ for different granular materials, ranging from sand, gravel, carbonate rocks up to cobbles (with $d_{50}$ ranging from 2.71 to $67.2 \mathrm{~mm}$ ). In the experimental study 
of Salahi et al. (2015), rounded and crushed angular grains (with $d_{50}$ ranging from 1.77 to $17.78 \mathrm{~mm}$ ) were used. The maximum Reynolds numbers used in these studies are 437-2709 (Moutsopoulos et al. 2009) and 714-1882 (Salahi et al. 2015).

It should be noted that the experiments for analyzing the onset of turbulence for flow in porous media (e.g., Seguin et al. 1998) are performed on spheres with one single diameter. However, natural granular materials have different grain size distributions, gradations and shape irregularities. The difference in pore structure and particle roughness could result in different Reynolds numbers for the onset of turbulent flow, and the critical Reynolds numbers for turbulence might significantly differ from 300 to 533. Andersen and Burcharth (1995) stated that for such materials the transition zones between the different flow regimes are blurred and difficult to define. This might explain that a reasonable singular fit with the Forchheimer equation (Eq. 2) is obtained for flow experiments on sand and gravel for a wide range of Reynold numbers (e.g., Moutsopoulos et al. 2009; Sedghi-Asl et al. 2014). Similar conclusions can be obtained from numerical studies, such as Firdaouss et al. (1997) and Fourar et al. (2004), which consider the Forchheimer equation (Eq. 2) valid over a wide range of Reynolds numbers.

Some studies have tried to characterize the nonlinear flow regime in terms of a weak inertial and strong inertial regime (fully turbulent flow regime) based on macroscale flow experiments in packed spheres of uniform size and determined different Forchheimer coefficients for both regimes (e.g., Fand et al. 1987; Bağci et al. 2014). The extent of these regimes was estimated from the reduced pressure drop versus flow velocity. Bağci et al. (2014) defined a turbulent flow regime for critical particle Reynolds numbers $>268$, while Fand et al. (1987) obtained Re values $>120$.

\subsection{Empirical Relations for Estimation of the Forchheimer Coefficients}

In many cases, the hydraulic characteristics by means of the Forchheimer coefficients $a$ and $b$ for a given porous medium sample are not available. Empirical relations can be used to predict the Forchheimer coefficients by using the soil characteristics, such as the average grain size and porosity. Many studies have developed empirical relations for the coefficients $a$ and $b$ (see Eq. 2). An overview of these expressions is given in Table 1.

Schneebeli (1955) proposed expressions for coefficients $a$ and $b$ for porous media consisting of spheres. The average particle diameter $(d)$ is used as characteristic pore length of the porous medium. Ward (1964) proposed similar expressions based on nonlinear flow experiments with 20 different porous media consisting of material ranging from glass beads to sand and gravel, having particle diameters of $0.27-16.1 \mathrm{~mm}$.

Based on the Kozeny-Carman relationship for Darcy flow (Carman 1937), Ergun (1952) derived an expression for the $b$ coefficient. The assumption of Ergun's (1952) approach is that fluid flow in porous media is similar to flow in pipes, assuming full turbulent flow in the nonlinear flow regime (Ergun 1952; Lage 1998). However, as described above, nonlinear flow at intermediate Reynolds numbers is laminar. Nevertheless, Ergun's (1952) relations work quite well for nonlinear flows in various kind of porous media and are, to a great extent, the most analyzed and used relationships (e.g., Sidiropoulou et al. 2007; Li and Ma 2011; Allen et al. 2013; Bağci et al. 2014). Many variations on the Ergun relations for different kind of porous media with a wide range of Ergun constants $A$ and $B$ are addressed in the literature (e.g., Macdonald et al. 1979; Fand et al. 1987; Comiti and Renaud 1989). Kovács (1981) analyzed a dataset of 300 sediment samples for $R e=10-100$ and proposed a value of 144 for constant $A$ and 2.4 for constant $B$. Du Plessis (1994) investigated the increase in flow resistance due to local flow recirculation in the pore spaces in the nonlinear laminar 
Table 1 Empirical relations for the Forchheimer coefficients $a$ and $b$

\begin{tabular}{llll}
\hline Equations & & Coefficient $a(\mathrm{~s} / \mathrm{m})$ & Coefficient $b\left(\mathrm{~s}^{2} / \mathrm{m}^{2}\right)$ \\
\hline Schneebeli (1955) & $(4)$ & $1100 \frac{v}{g d^{2}}$ & $12 \frac{1}{g d}$ \\
Ward (1964) & $(5)$ & $360 \frac{v}{g d^{2}}$ & $10.44 \frac{1}{g d}$ \\
Ergun-type & $(6)$ & $A \frac{(1-n)^{2} v}{g n^{3} d^{2}}$ & $B \frac{(1-n)}{g n^{3} d}$ \\
Carman (1937) & $A=180$ & - \\
Ergun (1952) & $A=150$ & $B=1.75$ \\
Kovács (1981) & $A=144$ & $B=2.4$ \\
Macdonald et al. (1979) & $(7)$ & $180 \frac{(1-n)^{2} v}{g n^{3.6} d^{2}}$ & $1.8 \frac{(1-n)}{g n^{3.6} d}$ \\
Kadlec and Knight (1996) & $(8)$ & $255 \frac{(1-n) v}{g n^{3.7} d^{2}}$ & $2 \frac{(1-n)}{g n^{3} d}$ \\
Sidiropoulou et al. (2007) & $(9)$ & $0.0033 d^{-1.50} n^{0.0603}$ & $0.194 d^{-1.27} n^{-1.14}$ \\
Geertsma (1974) & $(10)$ & - & $\left(\frac{0.005}{g}(k 10000)-0.5 n^{-5.5}\right)$ \\
\hline
\end{tabular}

Where $g$ is the acceleration due to gravity $\left(\mathrm{m} / \mathrm{s}^{2}\right), v$ is the kinematic viscosity $\left(\mathrm{m}^{2} / \mathrm{s}\right), d$ is the characteristic pore length by means of an average particle diameter $(\mathrm{m}), n$ is the porosity $(-)$, and $k(=\mu / \rho g a)$ is the intrinsic permeability $\left(\mathrm{m}^{2}\right)$

flow regime. From these analytical results, it was concluded that in the laminar flow regime the Ergun constants were higher than derived by Ergun (1952) for turbulent flow in porous media. Both constants are functions of porosity, where constant $A$ increases with increasing porosity, and constant $B$ decreases with increasing porosity. For porous media with a porosity ranging between 0.33 and 0.36 , constant $A$ is approximately 200 , while constant $B$ is 1.97 . Engelund (1953) derived a similar expression as Ergun (1952) for Forchheimer coefficient $b$ and stated that the constant $B$ varies from a value of 1.8 for uniform spherical particles, up to a value of 3.8 for highly irregular angular grains.

Macdonald et al. (1979) and Kadlec and Knight (1996) suggested different forms of the Ergun relation by changing the porosity term $(1-n)^{2} / n^{3}$ and $(1-n) / n^{3}$ for, respectively, coefficient $a$ and coefficient $b$ (see Table 1). Macdonald et al. (1979) used analyzed experimental datasets which included a wide variety of porous media (e.g., glass beads, steel spheres (blue metal), cylindrical fibers and granular material) with porosities ranging from 0.123 to 0.919. For Eq. $7 \mathrm{~b}$ of Macdonald et al. (1979), the constant 1.8 works well for smooth particles (see Table 1), while for rough particles a value of 4.0 is preferred for estimating the coefficient $b$. For intermediate surface roughness of the particles, the value should lie between these two values.

Sidiropoulou et al. (2007) derived empirical relationships, which are based on linear regression analysis on both porosity and the average particle size for a large dataset from the literature. Geertsma (1974) derived an empirical relationship for estimating Forchheimer coefficient $b$ based on intrinsic permeability, $k$, and porosity, examining an experimental dataset of consolidated and unconsolidated sandy porous media.

Many studies are using the median particle diameter $\left(d_{50}\right)$ as the characteristic length for the pore space. However, Macdonald et al. (1979) suggested that the use of the surfacevolume mean diameter $d_{S m}$ (also referred to as Sauter mean diameter) as a more appropriate characteristic length compared to the $d_{50}$. It is defined as the diameter of a sphere that has the same volume/surface area ratio as the particle of interest. For a perfect spherical particle, the surface area moment mean diameter $d_{S m}$ equals: 


$$
d_{S m}=6 \frac{V_{p}}{s_{p}}=d_{32}
$$

where $V_{p} / s_{p}$ is the ratio between volume and surface area of the sphere. Note that for nonspherical particles, as well as an rougher particles this ratio decreases and, hence, the average characteristic pore length of the porous medium decreases (Allen et al. 2013; Li and Ma 2011; Macdonald et al. 1979). In general, it is challenging to determine the exact surface area of natural porous materials.

\subsubsection{Evaluation of the Empirical Relations on Experimental Datasets of Granular Material}

Several studies have analyzed the theoretical and empirical relationships in Table 1 for Forchheimer coefficients $a$ and $b$. Sedghi-Asl et al. (2014) predicted the experimentally derived coefficients $a$ and $b$ accurately using Sidiropoulou et al. (2007) for sands with $d_{50}$ ranging from 2.83 to $56.8 \mathrm{~mm}$. A reasonable prediction for coefficient $a$ was obtained using Ergun (1952) and Kovács (1981). However, coefficient $b$ was systematically overestimated using Kovács (1981).

Moutsopoulos et al. (2009) predicted the experimentally derived coefficients $a$ and $b$ accurately using Kovács (1981) for sands with a $d_{50}$ of $2.71-67.23 \mathrm{~mm}$. The equation by Ward (1964) significantly underestimated the coefficients $a$ and $b$. The equation by Kadlec and Knight (1996) significantly overestimated coefficient $a$, but a good fit was obtained for the coefficient $b$. Moreover, Moutsopoulos et al. (2009) also suggested the use of $d_{20}$ and $d_{30}$ as particle diameter for the equation by Ward (1964), to provide good predictions for coefficients $a$ and $b$.

Sidiropoulou et al. (2007) investigated a large dataset collected from the literature. For coefficient $a$, the equation by Ward (1964) significantly underestimated the experimentally derived coefficient, while the equation by Kadlec and Knight (1996) resulted in significant overestimation of the coefficient. Only the equation by Ergun (1952), resulted in a reasonable fit for coefficients $a$ and $b$, where overall a underestimation was obtained for the experimentally derived coefficient. The equation by Ward (1964) resulted in significant underestimation of the values. Due to the relative poor fits, Sidiropoulou et al. (2007) suggested to use an alternative equation for accurate estimation of the Forchheimer coefficients (see Table 1). Salahi et al. (2015) also suggest the use of the equation proposed by Sidiropoulou et al. (2007) to obtain the best estimates for Forchheimer coefficients $a$ and $b$ for their dataset.

The purpose of the current study is to compare the expressions in Table 1 with our experimental dataset on filter sands and the experimental datasets in the literature.

\subsection{Friction Factor and Reynolds Number}

In the literature, many forms of the Darcy-Weisbach equation, which was initially derived for analyzing flow resistance in a conduit, are suggested for flow in porous media (e.g., Ergun 1952; Stephenson 1979; Herrera and Felton 1991). The method of Stephenson (1979) is widely used (e.g., Sedghi-Asl and Rahimi 2011; Salahi et al. 2015):

$$
f=\frac{2 i g d n^{2}}{q^{2}} \text { and } R e_{s}=\frac{\rho d_{50} q}{n \mu}
$$

The relation between Reynolds number $R e_{s}$ and the friction factor $f$ is usually given by:

$$
f=\frac{a_{s}}{R e_{s}}+b_{s}
$$


where $a_{s}$ and $b_{s}$ are dimensionless variables. Herrera and Felton (1991) used the mean grain size and standard deviation of the grains size distribution of a given sand type $\left(d_{m}-\sigma\right)$ as characteristic pore length in Darcy-Weisbach and Reynolds number equations (Eqs. 12, 13). They stated that for well-graded material with large standard deviations, finer particles fill the pores between coarser particles and the contact surface of particles with water increases. For such conditions, the flow resistance increases resulting in decreased flow velocity and Reynolds number. We have tested both the method of Stephenson (1979) with $\operatorname{Re}_{S}$ and Herrera and Felton (1991) with $R e_{H}$.

\section{Methods}

\subsection{Experimental Setup}

In order to measure one-dimensional flow in various sandy porous media, we used the experimental setup as shown in Fig. 1. It consists of the following main components; (1) the inlet, (2) the column with sandy porous medium, (3) the outlet and (4) the circulation tank of $1 \mathrm{~m}^{3}$ with a pump. A Plexiglas sample column (2) with a length of $0.507 \mathrm{~m}$, a wall thickness of $0.5 \mathrm{~cm}$ and an inner diameter of $9.8 \mathrm{~cm}$ contained the sandy porous media. The investigated sandy porous media have median grain sizes $\left(d_{50}\right)$ of $0.39-2.11 \mathrm{~mm}$ that are a factor 0.0040 and 0.0215 smaller than the diameter of the column, while for gravel $\left(d_{50}\right.$ of $\left.6.34 \mathrm{~mm}\right)$ the factor was 0.065 smaller. Fand and Thinakaran (1990) stated that the ratio between $d_{50}$ and column diameter should be lower than 0.025 , to ensure that the flow parameters are substantially independent of the wall effects of the column. The highest ratio in the literature for conducted flow experiments was in the same range (0.032 for Li et al. 2017, 0.089 for Salahi et al. 2015, 0.13 for Moutsopoulos et al. 2009 and 0.19 for Sedghi-Asl et al. 2014). The water pressure in the column was measured at a distance of $0.13,0.26$ and $0.38 \mathrm{~m}$ from the inlet. The pressure at the inlet compartment (1) was also measured, while at the outlet compartment (3) the hydraulic head was fixed at $0.505 \mathrm{~m}$. For all experiments, a linear hydraulic head gradient was obtained by interpolation of the measured water heads at the pressure monitoring points (5). The water pressure was measured with a BD Sensors pressure transmitter combined with a BD Sensors PA 430 display (5). The volumetric flow rate $Q\left(\mathrm{~m}^{3} / \mathrm{h}\right)$ was measured using a Metrima SVM F2 Energy Meter (6), which was placed between the pump (4) and the inlet compartment (1).

For each experiment on a single sand sample, the flow rate was determined for various hydraulic gradients, $i\left(=\left(h_{1}-h_{2}\right) / L\right)$, by using different injection pressures. The pressures ranged from 0.1 to 3.2 bar (15 different injection pressures) for the finest granular material (S.1) and ranged from 0.1 to 1.2 bar (12 different injection pressures) for the coarsest granular material (S.11). At a given hydraulic gradient, the flow rate was determined for at least more than 29 times to ensure achievement of steady state flow conditions (defined as $<3 \%$ variation in flow) in the porous medium. The temperature was measured in the outlet section of the experimental setup. To ensure constant water density and viscosity, the temperature in the circulation tank was kept constant at $20^{\circ} \mathrm{C}\left( \pm 0.3^{\circ} \mathrm{C}\right)$.

We have used the particle Reynolds number (Eq. 3) to determine the upper limit of the Darcy regime. For analysis, a deviation from Darcy's law is considered by observing a relative difference of $5 \%$ between the actual specific discharge described by the Forchheimer equation (Eq. 2) and the specific discharge described by Darcy's law (Eq. 1). The critical Reynolds numbers $\left(R e_{c r}\right)$ are obtained for all investigated sandy porous media. 


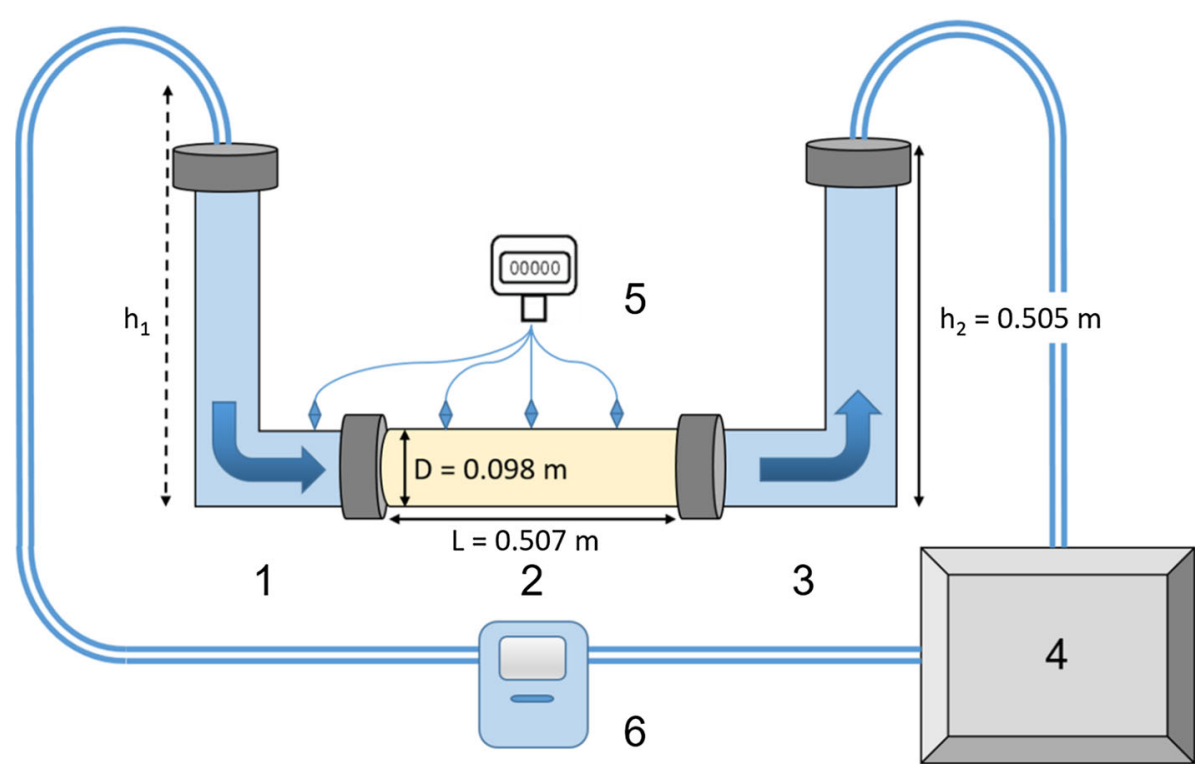

Fig. 1 Schematic overview of the experimental setup: 1 inlet compartment, 2 column with packed sandy porous medium, 3 outlet compartment, 4 circulating tank with pump, 5 pressure sensor and 6 volumetric flow meter

\subsection{Description of the Investigated Sandy Porous Media}

In this study, two sets of sandy porous media were investigated:

- At first, 11 uniformly graded filter sands (delivered by FILCOM company at Papendrecht, the Netherlands) with various median grain sizes $\left(d_{50}\right)$ were used as reference porous media to obtain the Forchheimer coefficients $a$ and bexperimentally (Samples 1-11). These filter sands are used as gravel pack around well screens to increase well efficiency, and also for packed sand beds for drinking water or wastewater treatment.

- Subsequently, these filter sands were mixed to obtain 9 different packed sandy porous media with wider grain size distributions at a given median grain size $\left(d_{50}\right)$ that equals one of the reference filter sands (Samples 3-8). Hence, we were able to test the effect of various grain size distributions for a given median grain size $\left(d_{50}\right)$ on nonlinear flow behavior experimentally (Samples 3.1, 4.1, 5.1 6.1-4, 7.1 and 8.1).

The porosity of each packed sample was determined by weighing the dry mass granular sand or gravel in the column. The solid-phase density of the sand and gravel equals $2.66 \mathrm{~kg} / \mathrm{m}^{3}$ (data by FILCOM).

The granulometric sieve data for the 11 reference sands were used to analyze the grain size distributions. The median grain size $\left(d_{50}\right)$, and the grain sizes $d_{10}, d_{30}$ and $d_{60}$ are listed in Table 2. The arithmetic method of moments was used to determine the mean particle diameter $\left(d_{m}\right)$ and the standard deviation $(\sigma)$ from the normal grain size distribution of the filter sands (Blott and Pye 2001), yielding

$$
d_{m}=\frac{\sum_{i=1}^{N} f_{i} d_{i}}{100}
$$


Table 2 The grain size distribution characterization for uniformly graded filter sands used as reference

\begin{tabular}{lllllllllll}
\hline Sample & $\begin{array}{l}d_{50} \\
(\mathrm{~mm})\end{array}$ & $\begin{array}{l}d_{10} \\
(\mathrm{~mm})\end{array}$ & $\begin{array}{l}d_{30} \\
(\mathrm{~mm})\end{array}$ & $\begin{array}{l}d_{60} \\
(\mathrm{~mm})\end{array}$ & $\begin{array}{l}d_{m} \\
(\mathrm{~mm})\end{array}$ & $\begin{array}{l}\sigma \\
(\mathrm{mm})\end{array}$ & $\begin{array}{l}d_{m}-\sigma \\
(\mathrm{mm})\end{array}$ & $C_{C}$ & $C_{U}$ & $n(-)$ \\
\hline 1 & 0.39 & 0.29 & 0.35 & 0.41 & 0.39 & 0.0845 & 0.31 & 1.05 & 1.39 & 0.344 \\
2 & 0.39 & 0.28 & 0.34 & 0.41 & 0.39 & 0.0852 & 0.30 & 1.04 & 1.48 & 0.335 \\
3 & 0.61 & 0.47 & 0.55 & 0.64 & 0.61 & 0.112 & 0.50 & 0.99 & 1.37 & 0.331 \\
4 & 0.71 & 0.53 & 0.63 & 0.76 & 0.72 & 0.155 & 0.57 & 0.99 & 1.42 & 0.340 \\
5 & 0.84 & 0.72 & 0.80 & 0.86 & 0.84 & 0.0921 & 0.75 & 1.02 & 1.19 & 0.333 \\
6 & 0.99 & 0.80 & 0.91 & 1.03 & 0.98 & 0.164 & 0.82 & 1.01 & 1.30 & 0.346 \\
7 & 1.05 & 0.89 & 0.98 & 1.08 & 1.05 & 0.138 & 0.91 & 1.00 & 1.21 & 0.348 \\
8 & 1.36 & 1.13 & 1.27 & 1.41 & 1.35 & 0.189 & 1.16 & 1.01 & 1.25 & 0.358 \\
9 & 1.50 & 1.16 & 1.34 & 1.58 & 1.52 & 0.287 & 1.23 & 0.98 & 1.36 & 0.358 \\
10 & 2.11 & 1.72 & 1.97 & 2.18 & 2.07 & 0.306 & 1.76 & 1.03 & 1.27 & 0.357 \\
11 & 6.34 & 5.16 & 5.87 & 6.55 & 6.28 & 0.938 & 5.34 & 1.02 & 1.27 & 0.361 \\
\hline
\end{tabular}

and

$$
\sigma=\sqrt{\frac{\sum_{i=1}^{N} f\left(d_{i}-d_{m}\right)^{2}}{100}},
$$

where $N$ is the number of used sieves for the grain size analysis, $f$ is the passing frequency in percent on the corresponding $i$ th sieve, and $d_{i}(\mathrm{~mm})$ is the average grain size diameter of the $i$ th sieve class interval. For the composite sands, specific portions of the reference sands were mixed. From the reference sand fractions, a new grain size distribution was determined and the $d_{m}$ and $\sigma$ were calculated based on a number of equal step intervals $(N)$ subdividing this grain size distribution. For non-uniformly graded granular material, Herrera and Felton (1991) used the standard deviation of particle sizes and substituted the characteristic pore length $d$ by $d_{m}-\sigma$.

Moreover, the coefficient of uniformity $C_{u}(-)$ and the coefficient of curvature $C_{c}(-)$ of the grain size distribution are calculated, using

$$
C_{U}=\frac{d_{60}}{d_{10}}
$$

and

$$
C_{C}=\frac{\left(d_{30}\right)^{2}}{d_{10} d_{60}}
$$

In line with the study of Moutsopoulos et al. (2009), we used the values $d_{10}, d_{30}$ and $d_{50}$ as characteristic pore length, $d$, in the empirical equations listed in Table 1. Also the method of Herrera and Felton (1991) was used by considering $d_{m}-\sigma$ as the characteristic pore length.

\subsubsection{Reference Sands}

The filter sands used in this study are classified as medium sand (S.1-2), coarse sand (S.3-6), very coarse sand (S.7-10) and fine gravel (S.11) (Blott and Pye 2001). The reference filter sands are uniformly graded with a $C_{u}<1.48, C_{c}$ ranging from 0.98 to 1.05 , and small arithmetic standard deviations $(\sigma<0.938 \mathrm{~mm}$ ) for all 11 samples (Table 2). All filter sands have a normal grain size distribution, resulting in approximately equal median grain sizes 
Table 3 The grain size distribution characterization for the filter sand mixtures

\begin{tabular}{lllllllllll}
\hline Sample & $\begin{array}{l}d_{50} \\
(\mathrm{~mm})\end{array}$ & $\begin{array}{l}d_{10} \\
(\mathrm{~mm})\end{array}$ & $\begin{array}{l}d_{30} \\
(\mathrm{~mm})\end{array}$ & $\begin{array}{l}d_{60} \\
(\mathrm{~mm})\end{array}$ & $\begin{array}{l}d_{m} \\
(\mathrm{~mm})\end{array}$ & $\begin{array}{l}\sigma \\
(\mathrm{mm})\end{array}$ & $\begin{array}{l}d_{m}-\sigma \\
(\mathrm{mm})\end{array}$ & $C_{C}$ & $C_{U}$ & $n(-)$ \\
\hline 3.1 & 0.62 & 0.40 & 0.53 & 0.66 & 0.62 & 0.168 & 0.45 & 1.06 & 1.63 & 0.334 \\
4.1 & 0.71 & 0.44 & 0.60 & 0.77 & 0.72 & 0.218 & 0.50 & 1.04 & 1.74 & 0.332 \\
5.1 & 0.84 & 0.57 & 0.73 & 0.89 & 0.84 & 0.203 & 0.64 & 1.06 & 1.56 & 0.337 \\
6.1 & 0.99 & 0.61 & 0.85 & 1.08 & 1.03 & 0.350 & 0.68 & 1.10 & 1.78 & 0.330 \\
6.2 & 0.99 & 0.68 & 0.89 & 1.03 & 0.96 & 0.208 & 0.75 & 1.12 & 1.52 & 0.344 \\
6.3 & 0.99 & 0.74 & 0.89 & 1.05 & 1.01 & 0.240 & 0.77 & 1.01 & 1.42 & 0.348 \\
6.4 & 1.01 & 0.53 & 0.74 & 1.23 & - & - & - & 0.84 & 2.32 & 0.332 \\
7.1 & 1.06 & 0.71 & 0.91 & 1.12 & 1.07 & 0.311 & 0.76 & 1.06 & 1.58 & 0.337 \\
8.1 & 1.36 & 0.81 & 1.12 & 1,49 & 1.39 & 0.453 & 0.93 & 1.03 & 1.84 & 0.345 \\
\hline
\end{tabular}

$\left(d_{50}\right)$ and mean grain sizes $\left(d_{m}\right)$. The roundness of the granular material is classified as subangular-subrounded.

\subsubsection{Composite Sands}

Table 3 and Fig. 9 show that the $d_{50}$ of the composite sands is equal to its corresponding reference sand. The composite sands (S.3.1-8.1) are slightly more well graded resulting in increased $C_{u}$ values by a factor of 1.19 up to 1.47 , while $C_{c}$ values are more or less equal to the reference sands. The wider grains size distribution is also reflected by the increased standard deviation values. All composite sand samples have a normal grain size distribution, except for Sample 6.4. For that case, a bimodal grain size distribution was obtained, with peaks at 0.75 and $1.38 \mathrm{~mm}$. Hence, a reduced $C_{c}$ value of 0.84 and an increased $C_{u}$ value of 2.32 are obtained.

The use of slightly more well-graded sands results in approximately equal porosities for both the reference and composite sands (with a relative errors ranging between 0.6 and $4.8 \%$ ). Much wider grains size distributions are required to obtain significant reduction of porosity at a given median grain size $\left(d_{50}\right)$. Only for the gap-graded composite sand S.6.4 with a clear bimodal grain size distribution, and for the more well-graded sand S.6.1 slightly lower porosity values of, respectively, 0.330 and 0.332 were obtained with respect to the reference sand S.6 $(n=0.345)$.

\subsection{Preparation Technique for the Sandy Porous Media}

A consistent and reproducible packing method for the column samples was used to obtain homogenously packed porous media. Different compaction preparation techniques were tested, to obtain the highest grain density and, hence, optimal compaction grades for each sample column. We followed the method by Rietdijk et al. (2010) by using a constant pluviation rate of the sand, as well a continuous manual compression of the deposited sand in a fully saturated column. This resulted in the lowest porosity values for the packed porous media samples, ranging from 0.33 for uniformly graded medium sand (Sample 1) to 0.36 for uniformly graded gravel (Sample 11) (Table 2). Other packing methods resulted in significantly higher porosities (up to $0.38-0.40$ ) of the porous medium. The lower compaction 
grades resulted in non-rigid pore structures at increased fluid pore pressures in the column during the flow experiment tests.

Repeatedly testing of the Rietdijk et al. (2010) method as sample preparation technique for a given filter sand showed that a reproducible compaction grade, and hence porosity (relative errors ranging between 0.02 and $1.1 \%$ ) was obtained. For flow behavior, the maximal relative error for coefficient $a$ was $2.0 \%$ and for coefficient $b 3.1 \%$. The reproducibility was tested for the reference filter sands S.2, S.3 and S.5. Therefore, we have used the Rietdijk et al. (2010) method for each sample to obtain an equal compaction grade and porosity over the entire column length and to ensure a stable pore structure of the sandy porous medium during the flow behavior experiments at increased injection pressures (up to 3 bar).

After the saturated sample preparation of the sample column inside a container filled with water, the inlet and outlet compartments were mounted under water to ensure no air inclusion occurred during the whole procedure. The water head at the inlet and outlet side provided fully saturated conditions during the installation into the experimental setup with the measuring equipment.

\subsection{Validity Analysis of the Empirical Relationships for Predicting Forchheimer Coefficients}

The experimental dataset from this study was used to analyze the validity of the empirical relationships listed in Table 1 . In order to compare the experimentally derived coefficients $a$ and $b$, with the empirically predicted coefficients by Eqs. 4-10, the following methods are used; (1) the normalized objective function (NOF) criterion and (2) linear regression analysis. A similar approach was used in the studies of Moutsopoulos et al. (2009), Sedghi-Asl et al. (2014) and Sidiropoulou et al. (2007). Hence, a comparison can be made between our results and the results of their studies.

The NOF is defined as the ratio between the root mean square error (RMSE) to the overall mean value $X$ of the experimental data.

$$
\mathrm{NOF}=\frac{\mathrm{RMSE}}{X},
$$

where:

$$
\operatorname{RMSE}=\sqrt{\frac{\sum_{i=1}^{N}\left(x_{i}-y_{i}\right)^{2}}{N}}
$$

and

$$
X=\frac{1}{N} \sum_{i=1}^{N} x_{i}
$$

Here $x_{i}$ are the experimental values for either the $a$ coefficients or the $b$ coefficients of the Forchheimer equation (Eq. 2) and $y_{i}$ the corresponding values computed by the empirical relationship (Eqs. 4-10) for which the NOF analysis is done. A NOF value lower than 1.0 implies a reasonable prediction of the coefficients $a$ and $b$ by the empirical relationships, where values close to 0.0 implies a perfect fit.

Linear regression is applied on scatter plots with the experimentally derived Forchheimer coefficient on the horizontal axis and the empirically predicted coefficient by the relationship (Eqs. 4-10) on the vertical axis. For a good prediction, the slope of the regression line equals 1.0. Values that are lower than 1.0, imply underestimation of the Forchheimer coefficients by the empirical relationship, while values higher than 1.0 imply overestimation. Moreover, 
the $R^{2}$-coefficient should be close to a value of 1.0 to ensure reduced scattering of the data points along the regression line.

\section{Results}

In this section, first, the reference experimental dataset on nonlinear flow behavior in the 11 samples of filter sands is presented. Subsequently, the nonlinear flow behavior in the composite sands is presented and compared to the reference dataset. Moreover, a comparison between the whole dataset of this study and the experimental data of existing literature is given. Finally, the empirical relationships for estimating the Forchheimer coefficients $a$ and $b$ in Table 1 (Eqs. 4-10) are verified using our experimental dataset.

\subsection{Nonlinear Flow Behavior in the Reference Sands}

Using the Forchheimer relation for nonlinear flow (Eq. 2) between the hydraulic gradient $i$ and the specific discharge $q$, the Forchheimer coefficients $a$ and $b$ are determined. Figure 2 shows the Forchheimer fits on the experimental data for all 11 reference sands. For all investigated samples, the nonlinear flow behavior could be accurately described using the Forchheimer relation, resulting in $R^{2}$-coefficients $>0.99$ (see Table 4). The linear Darcy flow $i=a q$ is also plotted (dashed line) to show the nonlinear deviation from Darcy's law at increased flow velocities. Table 4 shows that only Samples $1-4$ have a critical Reynolds number $R e_{c r}$ for the onset of nonlinear flow within the measured range $R e_{\min }-R e_{\max }$. The rest of the samples have only data in the nonlinear flow regime.

The critical Reynolds numbers in this study vary between 2.21 and 4.13 (see Table 4), which fall in the limits of 1-15 reported in the literature (e.g., Bear 1972; Hassanizadeh and Gray 1987; Comiti et al. 2000).

For the reference sands S.1 and 2, multiple data points were in the Darcy regime and, hence, the coefficient $a$ can be determined directly using a linear interpolation $\left(i=a_{\text {Darcy }} q\right)$. For Sample 1, coefficient $a_{\text {Darcy }}$ is 1962.5 with $R^{2}>0.99$ ( 5 data points), while for Sample 2, $a_{\text {Darcy }}$ is 1453.5 with $R^{2}=0.99$ ( 4 data points). The $a_{\text {Darcy }}$ coefficients are in agreement with the $a$ coefficients determined by the Forchheimer fit over the whole dataset (see Table 4), resulting in small relative errors of 2.1 and $1.9 \%$, respectively. This is in agreement with the results of Venkataraman and Rao (1998), who also observed similar coefficients for Forchheimer $a$ and $a_{\text {Darcy }}$. However, other studies showed that the Forchheimer coefficient $a$ in the nonlinear flow regime can significantly differ from the resistance coefficient $a_{\text {Darcy }}$ in the Darcy regime (see Bağci et al. 2014).

Note that the coefficient $a$ for S.2 is significantly lower than for S.1, while the grain size distribution and porosity of both filter sands are more or less identical. The decreased flow resistance for S.2 might be due to a significant difference in the geometry of the pore structure. However, such differences in geometry should be due to large contrast in particle roughness or grain shape, which is unlikely in our case with subangular-subrounded grains of the filter sands.

\subsection{Nonlinear Flow Behavior in the Composite Sands}

Similar to the reference sands, the nonlinear flow behavior for the composite sands could be described accurately by the Forchheimer equation (Eq. 2). The $R^{2}$-coefficients are larger than 0.99 for all plots (see Table 5). Figure 10 shows that for the slightly more well-graded 

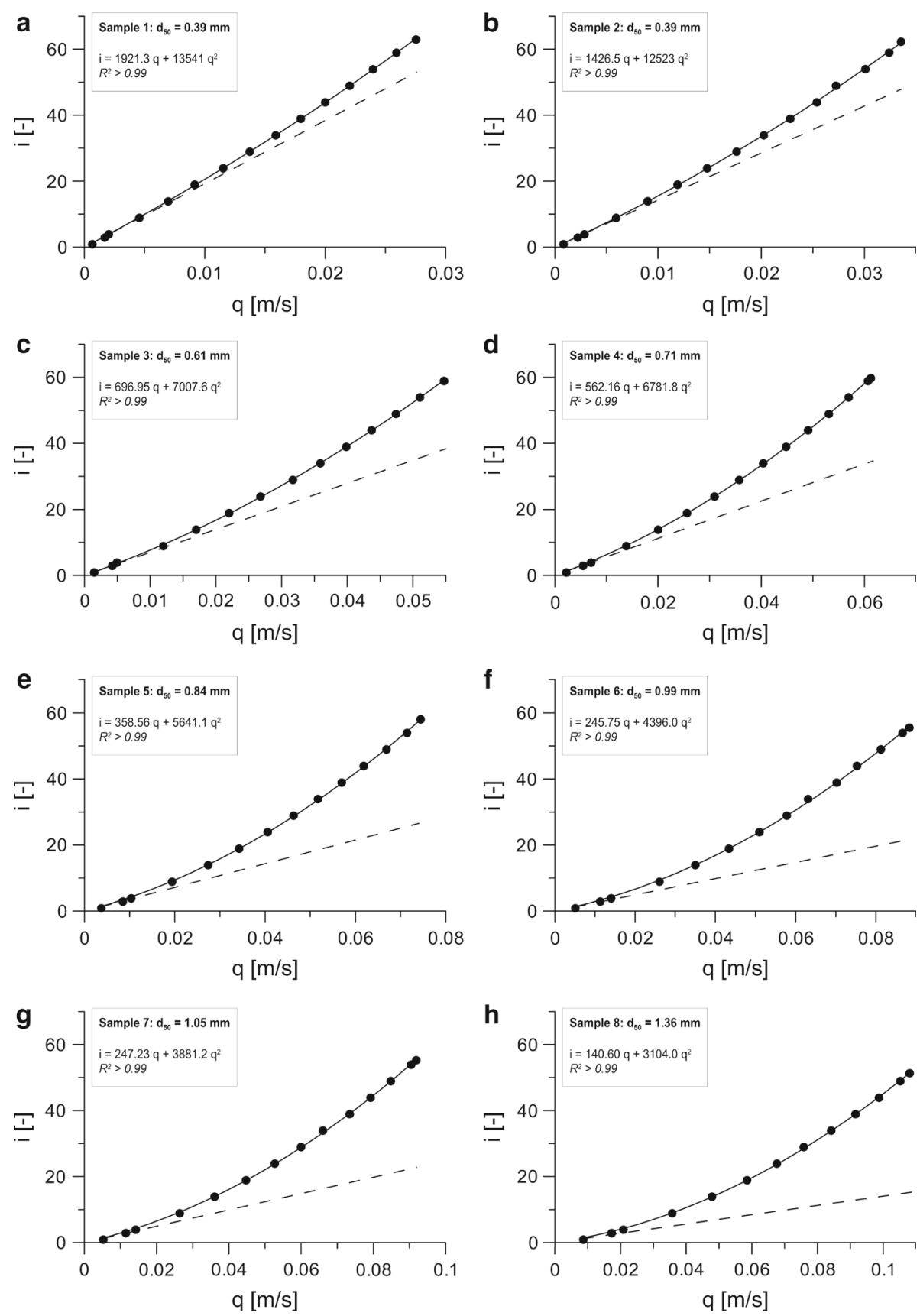

Fig. 2 The $i-q$ plots for the sandy reference porous media S1-11. The linear Darcy flow $i=a q$ is plotted (dashed line) to visualize nonlinear deviation from Darcy's law 

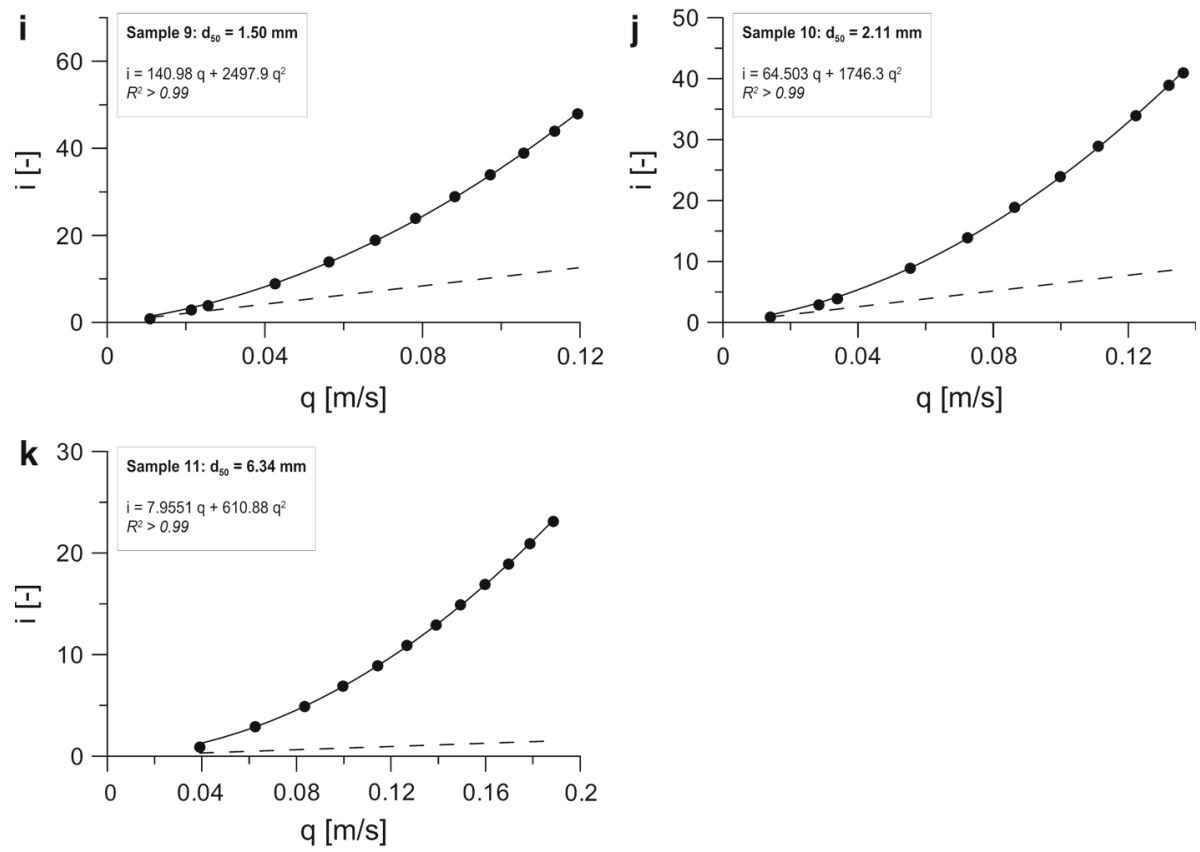

Fig. 2 continued

Table 4 The Forchheimer coefficients $a$ and $b$ for the reference sands (S.1-11)

\begin{tabular}{llrrrrrr}
\hline Sample & $d_{50}(\mathrm{~mm})$ & $R e_{\min }$ & $R e_{\max }$ & $R e_{c r}$ & $a\left(\mathrm{sm}^{-1}\right)$ & $b\left(\mathrm{~s}^{2} \mathrm{~m}^{-2}\right)$ & $R^{2}$ \\
\hline 1 & 0.39 & 0.26 & 10.72 & 2.76 & $1,921.3$ & 13,541 & $>0.99$ \\
2 & 0.39 & 0.33 & 13.02 & 2.21 & $1,426.5$ & 12,523 & $>0.99$ \\
3 & 0.61 & 1.21 & 34.59 & 2.67 & 696.96 & $7,007.6$ & $>0.99$ \\
4 & 0.71 & 1.58 & 43.72 & 2.96 & 562.16 & $6,781.8$ & $>0.99$ \\
5 & 0.84 & 3.28 & 62.07 & 2.69 & 358.56 & $5,641.1$ & $>0.99$ \\
6 & 0.99 & 5.02 & 87.29 & 2.76 & 245.75 & $4,396.0$ & $>0.99$ \\
7 & 1.05 & 5.51 & 96.28 & 3.34 & 247.23 & $3,881.2$ & $>0.99$ \\
8 & 1.36 & 11.78 & 147.06 & 3.08 & 140.60 & $3,104.0$ & $>0.99$ \\
9 & 1.50 & 16.33 & 179.44 & 3.16 & 104.98 & $2,497.9$ & $>0.99$ \\
10 & 2.11 & 29.65 & 287.92 & 3.90 & 64.503 & $1,746.3$ & $>0.99$ \\
11 & 6.34 & 248.34 & 1196.78 & 4.13 & 7.9551 & 610.88 & $>0.99$ \\
\hline
\end{tabular}

composite sands the flow resistance was increased due to an increase in the fraction of smaller particles (decreased $d_{10}$ and $d_{30}$ and increased $C_{u}$ values) with respect to the reference sands at equal median grain size $\left(d_{50}\right)$ (see Table 3 ; Figs. 3,10$)$. This means that the $a$ coefficients were increased by factors up to 1.68 , while the $b$ coefficients were increased by factors up to 1.44 with respect to the reference sands.

Four sands with different grain size distributions and similar $d_{50}$ compared to its associated reference sand S.6 were investigated (see Fig. 3b). Composite sand S6.3 shows a relative increase in coarser material (i.e., tailing in the grain size distribution) with respect to the 
Table 5 The Forchheimer coefficients $a$ and $b$ for the composite sands

\begin{tabular}{llrrrrrr}
\hline Sample & $d_{50}(\mathrm{~mm})$ & $R e_{\min }$ & $R e_{\max }$ & $R e_{c r}$ & $a\left(\mathrm{sm}^{-1}\right)$ & $b\left(\mathrm{~s}^{2} \mathrm{~m}^{-2}\right)$ & $R^{2}$ \\
\hline 3.1 & 0.617 & 0.97 & 32.38 & 2.67 & 740.18 & $8,219.7$ & $>0.99$ \\
4.1 & 0.713 & 1.44 & 40.29 & 2.84 & 623.57 & $7,839.1$ & $>0.99$ \\
5.1 & 0.841 & 2.46 & 58.25 & 2.77 & 418.33 & $6,349.4$ & $>0.99$ \\
6.1 & 0.993 & 3.36 & 73.71 & 3.24 & 362.31 & $5,561.2$ & $>0.99$ \\
6.2 & 0.988 & 3.86 & 77.58 & 3.11 & 326.26 & $5,181.8$ & $>0.99$ \\
6.3 & 0.99 & 5.51 & 86.67 & 2.90 & 256.80 & $4,380.2$ & $>0.99$ \\
6.4 & 1.006 & 3.08 & 70.14 & 3.27 & 412.74 & $6,351.4$ & $>0.99$ \\
7.1 & 1.048 & 4.44 & 84.89 & 3.19 & 283.51 & $4,965.6$ & $>0.99$ \\
8.1 & 1.363 & 10.16 & 138.27 & 3.26 & 168.36 & $3,518.9$ & $>0.99$ \\
\hline
\end{tabular}

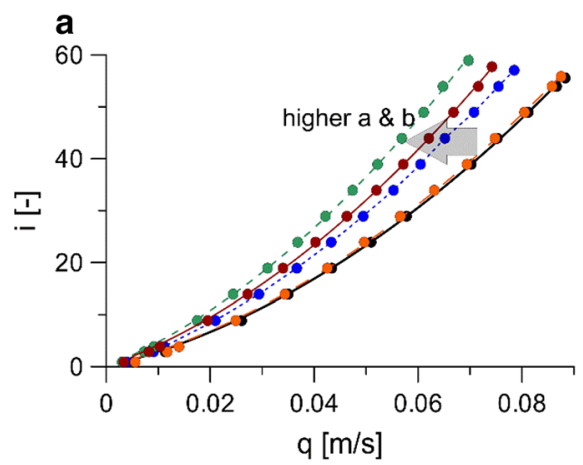

Sample 6 (ref.)

Sample 6.1

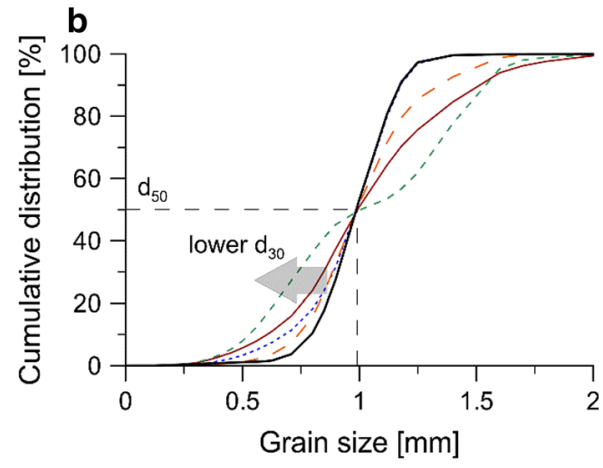

Sample 6.2

Sample 6.4

Fig. 3 a The $i-q$ plots and $\mathbf{b}$ the cumulative grain size distributions for the sandy porous media S6.1-4. Decreased $d_{30}$ results in increased flow resistance (higher $a$ and $b$ coefficients) compared to the reference sand S.6

reference sand S.6, while the finer material is equal. This resulted in approximately similar $a$ and $b$ coefficients for both S.6 and S.6.3. For the other composite sands S.6.2, 6.1 and 6.4 , the $d_{10}$ was decreased by a factor $0.86,0.76$ and 0.66 , respectively. This resulted in an increase by a factor $1.33,1.47$ and 1.68 for coefficient $a$ with respect to reference Sample 6 . For coefficient $b$, an increase by a factor $1.18,1.27$ and 1.44 was obtained. The bimodal grain size distribution of composite sand S.6.4 has a peak in the grain size distribution at $1.38 \mathrm{~mm}$. However, the relative increase in coarser material with respect to reference sand S.6 does not seem to influence the increase in flow resistance caused by the finer material.

\subsection{Comparison with the Literature}

In Fig. 4, the experimentally determined coefficients $a$ and $b$ are plotted against particle size $d_{50}$ for reference sands S.1-11, where a power function is fitted to the results. The Forchheimer coefficients increase with decreasing grain size of the porous media. The coefficients $a$ and $b$ for the composite sands are also plotted, but were not taken into account for fitting of the 


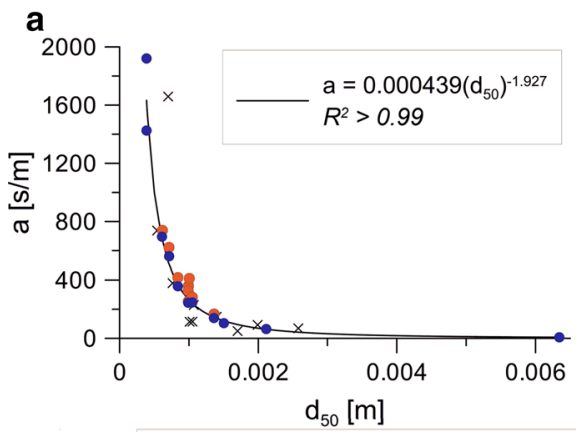

Ref. filter sands (Sample 1-11)

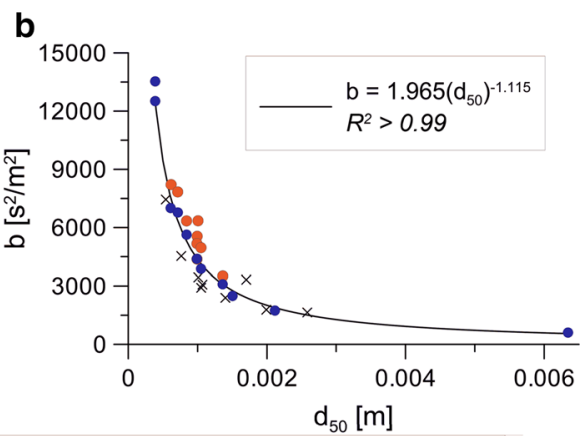

Composite sands

(Sample 3.1-8.1\&6.1-6.4)

Fig. 4 a Relationship between the median grain size $\left(d_{50}\right)$ and Forchheimer coefficient $a$, as well as for $\mathbf{b}$ Forchheimer coefficient $b$, for dataset Samples 1-11. The Forchheimer coefficients of the composite sands and sands provided by Sidiropoulou et al. (2007) with median grain sizes ranging from 0.7 to $2.58 \mathrm{~mm}$ are also plotted

power function. A poorer fit is obtained when the composite sand samples are taken into account. In this case, the $R^{2}$-coefficients are 0.98 and 0.97 for coefficient $a\left(=0.004 d^{-1.94}\right)$ and $b\left(=1.95 d^{-1.13}\right)$, respectively.

These results compare well to the data found by Sidiropoulou et al. (2007), who obtained the following relations; $a=0.00859 d^{-1.727}$ and $b=0.5457 d^{-1.253}$. Apart from Samples 10 and 11 , all other samples have a $d_{50}$ smaller than $2 \mathrm{~mm}$, while the dataset of Sidiropoulou is based on a broad data set of 89 samples with an average $d_{50}$ of $15 \mathrm{~mm}$. The Forchheimer coefficients provided by Sidiropoulou et al. (2007) for sands with median grain sizes $d_{50}$ ranging from 0.7 and $2.58 \mathrm{~mm}$ are selected and plotted in Fig. 4. For the samples with similar grain sizes in their dataset, the sample with the lowest porosity is used. Unfortunately, the porosity is not given for 8 of the samples, while for the other samples the porosity was significantly larger than in our experiments (with values of 0.381-392). This could explain the slightly lower values for the Forchheimer coefficients compared to our data, where the porosity values are significantly lower.

A large dataset on the intrinsic permeability $k\left(\mathrm{~m}^{2}\right)$ of sands is available in the literature, while in most cases the nonlinear term $b$ of the Forchheimer is unknown. Hence, we have plotted the Forchheimer coefficient $a$, which is equal to $\mu / \rho g k$ (e.g., Venkataraman and Rao 1998), as a function of coefficient $b$ for all samples (see Fig. 5) where a good correlation between both values is found. This function could be used to estimate the Forchheimer coefficient $b$ from experimental values of the intrinsic permeability in the literature for similar subangular-subrounded uniformly graded sand types.

Our dataset is compared to the recent studies of Sidiropoulou et al. (2007), Moutsopoulos et al. (2009), Bağci et al. (2014), Sedghi-Asl et al. (2014), Salahi et al. (2015) and Li et al. (2017). The Moutsopoulos et al. (2009), Salahi et al. (2015) and Li et al. (2017) datasets on coarser granular material correspond well to the fit on our dataset of finer sands (see Fig. 5). The flow experiments in these studies are conducted at particle Reynold numbers in the same range as in our study (up to $R e=560.97$ for Moutsopoulos et al. (2009), up to $R e=1882$ for Salahi et al. (2015) and up to $R e=40$ for Li et al. (2017). The same applies to Bağci et al. (2014), where nonlinear flow in packed porous media of mono-size steel balls is investigated. From the Sidiropoulou et al. (2007) dataset, the data for granular sands, gravel and round river gravel by Ahmed and Sunada (1969) (data by Ahmed); Ranganadha Rao and Suresh (1970), 

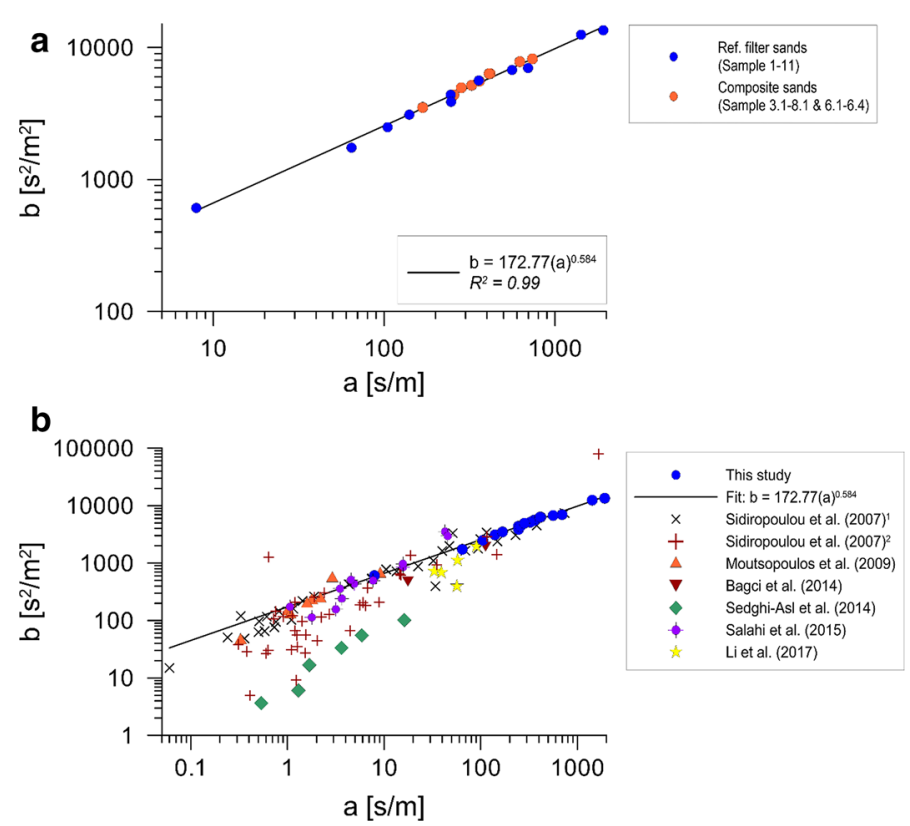

Fig. 5 a Forchheimer coefficient $b$ as a function of coefficient $a$ for the reference and composite sands. b Forchheimer coefficient $b$ versus coefficient $a$ for literature datasets. 1) Data from Sidiropoulou et al. (2007) Ahmed and Sunada (1969) (data by Ahmed), Ranganadha Rao and Suresh (1970), Tyagi and Todd (1970) and Arbhabhirama and Dinoy (1973). 2) Data from Sidiropoulou et al. (2007) by Ahmed and Sunada (1969), Venkataraman and Rao (1998) and Bordier and Zimmer (2000)

Tyagi and Todd (1970) and Arbhabhirama and Dinoy (1973) correspond well. Sedghi-Asl et al. (2014) and the rest of the dataset of Sidiropoulou et al. (2007) (Ahmed and Sunada 1969; Venkataraman and Rao 1998; Bordier and Zimmer 2000), which mainly consists of glass spheres and crystalline rock, underestimate $b$ for a given $a$, compared to our dataset.

Similar to the current study, the effect of the grain size distribution on nonlinear flow behavior was shown by Moutsopoulos et al. (2009) for different mixtures of quarry carbonate rocks. The grain size $d_{10}-d_{60}$ values of the mixtures are shown in Fig. 6b. The reference material M.3 has approximately the same median grain size $\left(d_{50}\right)$ as the composite material M.7. Similar to the present study, increased fraction of finer material (decreased value of $d_{30}$ ) for M.7 results in increased flow resistance of the porous media.

\subsection{Analysis of the Relationships for Predicting Forchheimer Coefficients}

The NOF criterion and the linear regression method (mentioned in Sect. 3.4) are used to evaluate the reliability of the empirical relationships provided in Table 1 . The entire dataset of both reference and composite sands is used for the analysis. Considering the median grain size $\left(d_{50}\right)$, the relationships of Ergun (1952), Macdonald et al. (1979) and Kovács (1981) seem to provide the most accurate estimation for coefficient $a$ with regression slopes of 1.48, 0.64 and 0.62, respectively (Table 6). For coefficient $b$, Macdonald et al. (1979) and Kovács (1981) obtained the best fits, resulting in a regression slope of 1.20 and 0.84 , respectively (see Table 7). The accuracy of Macdonald et al. (1979) and Kovács (1981) is also confirmed by the NOF analysis (see Tables 6, 7). Overall, the relationship of Macdonald et al. (1979) overestimates the flow resistance, while Ergun (1952) and Kovács (1981) underestimate the 

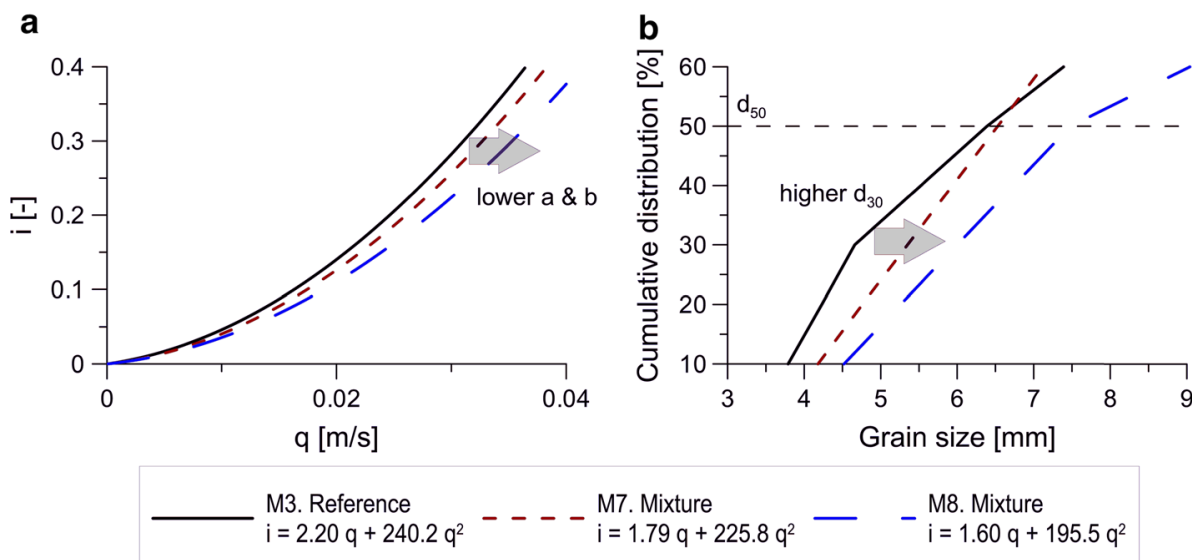

Fig. 6 a Forchheimer $i-q$ plot for different mixtures of quarry carbonate rocks and $\mathbf{b}$ the associated grain size distributions (obtained from Moutsopoulos et al. 2009). Increased $d_{30}$ results in decreased flow resistance (lower $a$ and $b$ coefficients) compared to the reference material M3

flow resistance, using the median grain size $\left(d_{50}\right)$. The relationship by Sidiropoulou et al. (2007) seems to be reliable for the estimation of the coefficient $b$ using $d_{50}$, while a poor fit was obtained for coefficient $a$. In order to investigate the empirical relation of Geertsma (1974), the intrinsic permeability was calculated from the determined $a$ coefficient from our experimental dataset. A poor estimation of the coefficient $b$ was obtained.

Generally, the use of the characteristic pore length $d_{m}-\sigma$ in the empirical relationships (Eqs. 4a-9a) results in the best estimates for coefficient $a$, except for Macdonald et al. (1979) and Kadlec and Knight (1996). Macdonald et al. (1979) also results in poor estimated for coefficient $b$. For both Ergun (1952) and Kovács (1981), the best estimations for both coefficient $a$ and coefficient $b$ are obtained. Also, the use of $d_{10}$ results in reasonable regression slope values. However, for the estimation of coefficient $a$ while using $d_{10}$, the data are more scattered around the regression line, resulting in a $R^{2}$-coefficient of 0.89 , while 0.94 is obtained for $d_{m}-\sigma$.

\subsubsection{Derivation of Ergun Constants Based on the Experimental Dataset}

The Ergun constants $A$ and $B$ are determined for our dataset following the linear regression procedure (mentioned in Sect. 3.4). This is done for the median grain size $\left(d_{50}\right)$, as well as for $d_{10}, d_{30}$ and $d_{m-\sigma}$. Using the median grain size $\left(d_{50}\right)$, or $d_{30}$, the Ergun constants based on our experimental dataset are higher than the values provided by Ergun (1952) and Kovács (1981) (Table 8).

In the literature, a wide range of different values of the Ergun constants can be found. According to Du Plessis (1994) analytical study on flow behavior in the nonlinear laminar flow regime, higher constants $A$ and $B$ in the range of 200 and 1.97 should be used. The Ergun constant $B$ of $2.88\left(d_{50}\right)$ and $2.53\left(d_{30}\right)$ is in agreement with the value provided by Engelund (1953), who stated that a value of approximately 2.8 should be used for uniform and rounded sand grains. The range of typical suitable experimental Ergun constants for sands and gravel in the literature is large. The dataset of sand and gravel in Macdonald et al. (1979) provides an average Ergun constant $A$ of 254.4 (range of 142-488) and an average Ergun constant of 2.024 (ranging from 2.52 to 1.01). Analysis of the Li et al. (2017) dataset results in an average Ergun constant $A$ of 349.6 and an average Ergun constant $B$ of 1.786. 
Table 6 Linear regression slope and NOF value for the evaluation of the empirical relationships for the Forchheimer coefficient $a$

\begin{tabular}{|c|c|c|c|c|c|c|c|c|c|c|c|c|}
\hline & \multicolumn{3}{|l|}{$d_{10}$} & \multicolumn{3}{|l|}{$d_{30}$} & \multicolumn{3}{|l|}{$d_{50}$} & \multicolumn{3}{|c|}{$d_{m}-\sigma$} \\
\hline & Slope & $R^{2}$ & NOF & Slope & $R^{2}$ & $\mathrm{NOF}$ & Slope & $R^{2}$ & $\mathrm{NOF}$ & slope & $R^{2}$ & NOF \\
\hline $\begin{array}{l}\text { Schneebeli (1955) } \\
\quad E q .4 a\end{array}$ & 0.808 & 0.93 & 0.341 & 0.530 & 0.95 & 0.664 & 0.418 & 0.96 & 0.815 & 0.701 & 0.96 & 0.438 \\
\hline Ward (1964) Eq. $5 a$ & 0.265 & 0.93 & 1.026 & 0.174 & 0.95 & 1.151 & 0.137 & 0.96 & 1.202 & 0.229 & 0.96 & 1.074 \\
\hline $\begin{array}{l}\text { Ergun (1952) Eq. 6a } \\
\quad \text { with } A=150\end{array}$ & 1.128 & 0.89 & 0.537 & 0.816 & 0.93 & 0.340 & 0.643 & 0.94 & 0.525 & 1.078 & 0.94 & 0.295 \\
\hline $\begin{array}{l}\text { Kovács (1981) Eq. } 6 a \\
\quad \text { with } A=144\end{array}$ & 1.198 & 0.89 & 0.482 & 0.783 & 0.93 & 0.370 & 0.617 & 0.94 & 0.557 & 1.035 & 0.94 & 0.268 \\
\hline $\begin{array}{l}\text { Macdonald et al. } \\
\text { (1979) Eq. } 7 a\end{array}$ & 2.871 & 0.89 & 2.782 & 1.877 & 0.92 & 1.332 & 1.477 & 0.94 & 0.774 & 2.480 & 0.93 & 2.161 \\
\hline $\begin{array}{l}\text { Kadlec and Knight } \\
\text { (1996) Eq. } 8 a\end{array}$ & 4.533 & 0.89 & 5.158 & 2.963 & 0.92 & 2.861 & 2.332 & 0.93 & 1.959 & 3.914 & 0.93 & 4.189 \\
\hline $\begin{array}{l}\text { Sidiropoulou et al. } \\
\text { (2007) Eq. 9a }\end{array}$ & 0.425 & 0.86 & 0.811 & 0.309 & 0.90 & 0.965 & 0.258 & 0.91 & 1.035 & 0.381 & 0.91 & 0.867 \\
\hline
\end{tabular}

Table 7 Linear regression slope and NOF value for the evaluation of the empirical relationships for the Forchheimer coefficient $b$

\begin{tabular}{|c|c|c|c|c|c|c|c|c|c|c|c|c|}
\hline & \multicolumn{3}{|l|}{$\mathrm{d}_{10}$} & \multicolumn{3}{|l|}{$\mathrm{d}_{30}$} & \multicolumn{3}{|l|}{$\mathrm{d}_{50}$} & \multicolumn{3}{|c|}{$\mathrm{d}_{m}-\sigma$} \\
\hline & Slope & $\mathrm{R}^{2}$ & NOF & Slope & $\mathrm{R}^{2}$ & NOF & Slope & $\mathrm{R}^{2}$ & NOF & Slope & $R^{2}$ & $\mathrm{NOF}$ \\
\hline $\begin{array}{l}\text { Schneebeli (1955) } \\
\quad E q .4 b\end{array}$ & 0.345 & 0.97 & 0.746 & 0.277 & 0.97 & 0.823 & 0.244 & 0.95 & 0.861 & 0.317 & 0.98 & 0.776 \\
\hline $\begin{array}{l}\text { Ward (1964) } \\
\text { Eq. } 5 b\end{array}$ & 0.300 & 0.97 & 0.797 & 0.241 & 0.97 & 0.864 & 0.212 & 0.95 & 0.897 & 0.277 & 0.98 & 0.826 \\
\hline $\begin{array}{l}\text { Ergun (1952) } \\
\quad \text { Eq. } 6 b \text { with } B=1.75\end{array}$ & 0.865 & 0.95 & 0.188 & 0.694 & 0.96 & 0.357 & 0.610 & 0.96 & 0.450 & 0.797 & 0.97 & 0.243 \\
\hline $\begin{array}{l}\text { Kovács (1981) } \\
\quad E q .6 b \text { with } B=2.4\end{array}$ & 1.186 & 0.95 & 0.258 & 0.951 & 0.96 & 0.115 & 0.836 & 0.96 & 0.209 & 1.093 & 0.97 & 0.148 \\
\hline $\begin{array}{l}\text { Macdonald et al. } \\
\text { (1979) Eq. } 7 b\end{array}$ & 1.707 & 0.94 & 0.836 & 1.368 & 0.96 & 0.447 & 1.2035 & 0.95 & 0.270 & 1.572 & 0.96 & 0.672 \\
\hline $\begin{array}{l}\text { Kadlec and Knight } \\
\text { (1996) Eq. } 8 b\end{array}$ & 0.988 & 0.95 & 0.123 & 0.793 & 0.96 & 0.250 & 0.697 & 0.96 & 0.354 & 0.911 & 0.97 & 0.134 \\
\hline \multirow{3}{*}{$\begin{array}{l}\text { Sidiropoulou et al. } \\
\text { (2007) Eq. } 9 b\end{array}$} & 1.480 & 0.96 & 0.578 & 1.122 & 0.96 & 0.198 & 0.955 & 0.94 & 0.154 & 1.337 & 0.97 & 0.414 \\
\hline & & & & \multicolumn{9}{|c|}{$k(=\mu / \rho g a)$} \\
\hline & & & & Slope & & & & $R^{2}$ & & & & $\mathrm{NOF}$ \\
\hline $\begin{array}{l}\text { Geertsma (1974) Eq. } \\
\quad 10 b\end{array}$ & & & & 0.2068 & & & & 0.95 & & & & 0.904 \\
\hline
\end{tabular}

From the Sidiropoulou et al. (2007) dataset, the data for granular sands, gravel and round river gravel by Arbhabhirama and Dinoy (1973), as well as Ranganadha Rao and Suresh (1970), are used, resulting in an average Ergun constant $A$ of 257.6 and an average Ergun constant $B$ of 2.89. For the dataset of Moutsopoulos et al. (2009) an average Ergun constant 
Table 8 Estimation of the Ergun constant $A$ and $B$ with linear regression analysis at $\gamma=1.00$ using all sand samples

\begin{tabular}{llllll}
\hline Particle size & Ergun constant $(E q .7)$ & Slope & $R^{2}$ & NOF \\
\hline$d_{50}$ & A & 233.5 & 1.00 & 0.94 & 0.260 \\
& B & 2.88 & 1.00 & 0.96 & 0.113 \\
$d_{10}$ & A & 120.3 & 1.00 & 0.89 & 0.330 \\
& B & 2.03 & 1.00 & 0.94 & 0.124 \\
$d_{30}$ & A & 183.8 & 1.00 & 0.93 & 0.275 \\
& B & 2.53 & 1.00 & 0.96 & 0.106 \\
$d_{m}-\sigma$ & A & 139.1 & 1.00 & 0.94 & 0.254 \\
& B & 2.20 & 1.00 & 0.97 & 0.095 \\
\hline
\end{tabular}

$A$ of 220.4 (range of 144.7-429.2) is derived using $d_{50}$, while the derived Ergun constant $A$ for cobbles is erroneously high (value of 1963). The average Ergun constant $B$ is 2.26 (range of 1.95-3.39). The Ergun constants provided by these studies on nonlinear flow behavior in granular material compare well to the values we have obtained in this study.

The use of a smaller grain size $d_{30}$, as well as $d_{m}-\sigma$, for the characteristic pore length, and their associated derived Ergun constants, obtained approximately similar NOF values and $R^{2}$-coefficients for the regression line (see Table 8 ) as compared to fitting analysis using $d_{50}$. The fits using $d_{10}$ are less accurate.

\subsubsection{Ergun Constants and the Effect of Finer Material}

In order to investigate the effect of the different grain size distributions of the composite sands with respect to the reference sands, linear regression analysis is applied to the reference sands S.3-8 and the corresponding sand mixtures (see Fig. 7; Table 9). The Ergun constants derived from the entire dataset of this study were used (see Table 8). The use of $d_{30}$ and $d_{m}-\sigma$ and associated Ergun constants $A$ and $B$ results in only a slightly better fit with the experimental Forchheimer coefficients. It should be noted that small variations in porosity between the reference and composite sands (see Tables 2,3) affect the prediction of coefficient $a$ and coefficient $b$ using equation 6 . Note that the $d_{30}$ for the composite sands is decreased by factors ranging between 0.81 up to only 0.96 . Hence, the characteristic pore length of $d_{30}$ might provide better estimations for the Forchheimer coefficients for well-graded sands with a significant increase in finer material (significantly decreased $d_{30}$ values) with respect to uniformly graded sands at approximately equal porosity values. The same holds for the use of $d_{m}-\sigma$. However, compaction of typical well-graded sandy soils with wide grain size distributions normally results in reduced porosity values compared to uniformly graded sands.

\subsection{Friction Factor}

The friction factor calculated with Eq. (12) is plotted versus the Reynolds number $R e_{s}$ in Fig. 8. We used regression analysis on our full dataset to obtain the following relationship:

$$
f=\frac{1340}{R e_{s}}+19.35 ; R>0.96
$$

At Reynolds numbers $R e_{s}$ higher than 9000, $f<19.5$. This means that in this case Forchheimer equation reduces to $b q^{2}$ and flow can be considered fully turbulent (Andersen and Burcharth 1995; Venkataraman and Rao 1998; Salahi et al. 2015). Our results are in agree- 


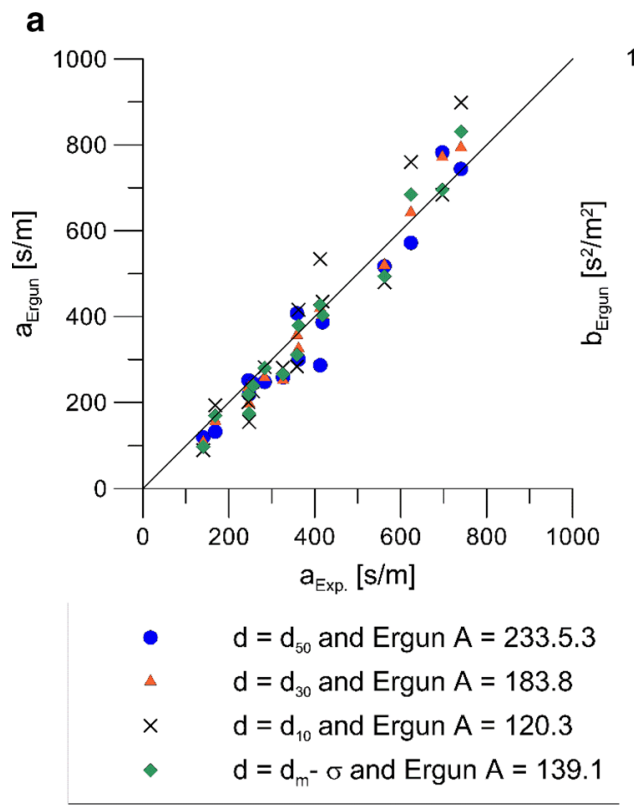

b

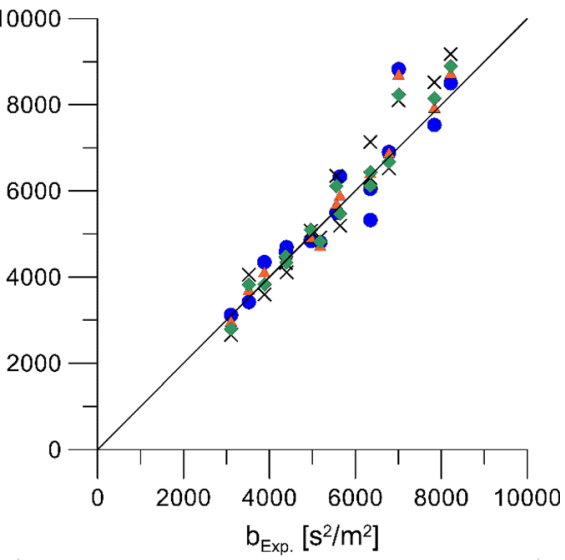

- $\quad \mathrm{d}=\mathrm{d}_{50}$ and Ergun $\mathrm{B}=2.88$

4 $\quad d=d_{30}$ and Ergun $B=2.53$

$\times \quad d=d_{10}$ and Ergun $B=2.03$

$\checkmark \quad d=d_{m}-\sigma$ and Ergun $B=2.2$

Fig. 7 a Analysis of the reference sands (S.3-8) and all composite sands using the relationship of Ergun (1952) (see Eq. 6) for Forchheimer coefficient $a$, and b Forchheimer coefficient $b$

Table 9 Analysis of the reference sands (S.3-8) and all composite sands using the relationship of Ergun (1952) with the Ergun constants provided in Table 8

The used data are plotted in Figure 7

\begin{tabular}{lllll}
\hline Particle size & \multicolumn{2}{l}{ Ergun constant (Eq. 6) } & Slope & $R^{2}$ \\
\hline$d_{50}$ & A & 233.5 & 1.0502 & 0.89 \\
& B & 2.88 & 1.0464 & 0.93 \\
$d_{10}$ & A & 120.3 & 0.9595 & 0.96 \\
& B & 2.03 & 1.02 & 0.86 \\
$d_{30}$ & A & 183.8 & 1.0021 & 0.97 \\
& B & 2.53 & 1.032 & 0.93 \\
$d_{m}-\sigma$ & A & 139.1 & 1.0001 & 0.95 \\
& B & 2.20 & 1.032 & 0.95 \\
\hline
\end{tabular}

ment with Salahi et al. (2015), who obtained $f=\frac{967}{R e_{s}}+9.45$ with a $R^{2}$ of 0.98 for their experimental dataset on coarse granular material.

We have also followed the method of Herrera and Felton (1991) to relate the friction factor to their proposed Reynolds number $\operatorname{Re}_{H}$ :

$$
f=\frac{822.3}{R e_{H}}+13.16 ; R^{2}>0.95
$$

Herrera and Felton (1991) proposed their form of the Darcy-Weisbach equation for wellgraded coarse material. However, the composite sands in our dataset are only slightly more well-graded compared to our reference dataset $\left(C_{u}<1.8\right)$. Hence, no large difference between the method of Stephenson (1979) and Herrera and Felton (1991) is found for our dataset. 
Fig. 8 The relationship between Reynolds number $\operatorname{Re}_{S}$ and friction factor $f$ using the Stephenson (1979) method

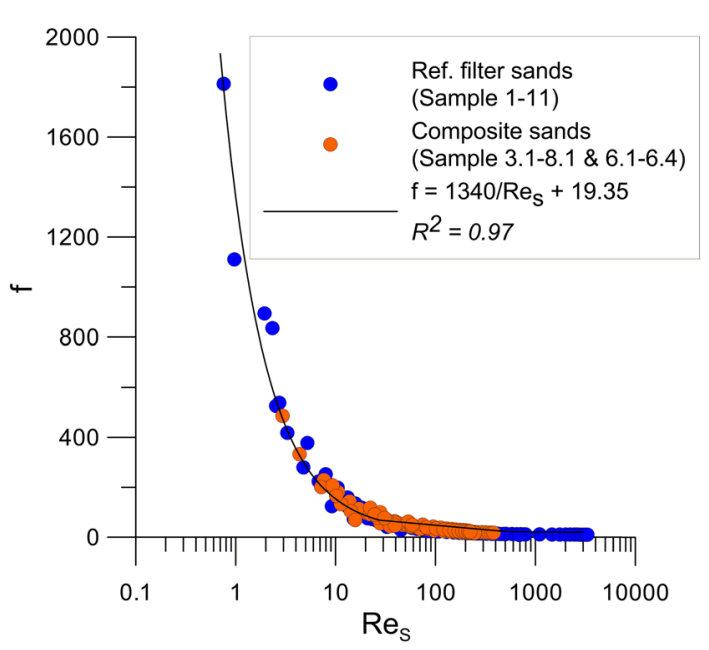

\section{Discussion}

In this study, nonlinear flow behavior experiments on the composite and the reference sands (S.1-9) were conducted at minimum particle Reynold numbers ranging between 0.26 and 16.33 (Tables 4, 5). Hence, it is likely that the majority of the flow dataset at lower Reynold numbers is determined at the onset of the nonlinear laminar flow regime. Experimental studies on flow behavior in packed uniform spheres determined critical Reynolds numbers ( $R e>300$ ) for fully turbulent flow (e.g., Dybbs and Edwards 1984; Seguin et al. 1998). According to Andersen and Burcharth (1995), it is challenging to define the transition from nonlinear laminar flow to fully turbulent flow for granular, irregular shaped, porous media. Consequently, it is difficult to identify to what extent the fully turbulent flow is developed at increased particle Reynolds numbers in the coarser material. Overall, the grain sizes of the investigated sands in this study are significantly lower than for the sands and gravel in the literature, and therefore, the observed flow resistance by means of Forchheimer coefficients $a$ and $b$ is significantly higher (see Fig. 5b).

To date, complete understanding of the macroscopic nonlinear flow behavior at specific Reynold numbers for different complex pore structures is hampered due to insufficient knowledge about the link between the macroscopic flow characteristics and complex microscopic pore structures. Many attempts have been made to link Forchheimer coefficients $a$ and $b$, to different pore scale parameters. For example, some studies have linked the effect of the tortuosity (ratio between effective hydraulic stream line length and straight-line length between two points to characterize fluid pathways) to the Forchheimer coefficient $b$ (e.g., Liu et al. 1995; Thauvin and Mohanty 1998). Overall, increased tortuosity of a porous medium results in increased flow resistance and, therefore, larger $b$ coefficients. However, it is difficult to obtain the tortuosity for complex pore structures of granular porous media. Moreover, as described in Sect. 2.2, drag forces that determine the nonlinear laminar flow in porous media are controlled by the fluid-solid interfaces of the pore structures and pore geometry (e.g., Hassanizadeh and Gray 1987; Comiti et al. 2000; Panfilov and Fourar 2006). Hence, it seems reasonable to characterize the porous media by means of the surface area and its roughness. For example, the capillary representation model of Comiti et al. (2000) used a dynamic 
surface area of the porous media, as well as the tortuosity, while assuming a cylindrical characterization of the pores.

The results in Sect. 4.2 show that an increased fraction of finer material at fixed values of $d_{50}$ and porosity results in an increased flow resistance. Under this condition, the use of the characteristic pore length by means of the grain size diameter $d_{30}$ in the modified Ergun relationships works well for accurate prediction of the Forchheimer coefficients $a$ and $b$ to account for finer material.

As mentioned earlier, Macdonald et al. (1979) suggest the use of the Sauter mean (Eq. 11) as a measure of the characteristic pore length in the relationships (Eqs. 6, 7). This definition of the characteristic pore length emphasizes the importance of specific surface area in complex pore structures. Hence, it seems sensible that the nonlinearity in flow resistance due to interfacial drag forces acting on the fluid-solid interface should be upscaled to a macroscopic parameter that characterizes pore surface area. For grain size distributions considering perfect spherical grains, the $d_{32}$ can be used. However, for more angular materials with lower sphericity values, the ratio between volume and surface area is lowered and smaller characteristic pore lengths should be considered. In our study, the $d_{30}$ of uniformly graded sand approximately equals the Sauter mean, considering rounded sand grains. Herrera and Felton (1991) suggested to use the standard deviation of particle sizes and substituted the characteristic pore length $d$ by $d_{m}-\sigma$. For more well-graded sand types, with larger standard deviations, finer particles fill the pores between coarser particles and the contact surface of particles with water increases. Tables 2 and 3 show that the characteristic pore length of $d_{m}-\sigma$ is smaller than $d_{30}$ and, hence, accounts more for the finer particles in the packed-column sample.

Nonetheless, it should be noted that fitting of nonlinear flow data with the Forchheimer relation (Eq. 2) over a wide range of Reynold numbers describes the gradual macroscopic transition from laminar Darcy flow to nonlinear laminar flow, and, eventually, to fully turbulent flow. Hence, linking between the different macroscopic flow characteristics at a wide range of Reynold numbers and the complex microscopic pore structures by means of , e.g., characteristic pore length, tortuosity, compaction grade, sphericity is complicated.

\section{Conclusions}

The current study provides new experimental data on nonlinear water flow behavior in various uniformly graded granular material for 20 samples, ranging from medium sands $\left(d_{50}>0.39\right.$ $\mathrm{mm})$ to gravel $(6.34 \mathrm{~mm})$. The effect of the grain size distribution on the macroscopic flow resistance parameters is analyzed using the Forchheimer coefficients. As the reference dataset, we have used 11 uniformly graded filter sands. In addition, the mixtures of the filter sands are used to obtain composite sands with different grain size distributions. This provided samples with increased $C_{u}$ values by a factor of 1.19 up to 2.32 at equal median grain size $d_{50}$ and porosity relative to the associated reference sands. The main conclusions from this study are:

- Our dataset has shown that the $d_{50}$ value is not enough to predict flow resistance accurately. Wider grain size distributions, indicated by an increase in $d_{10}$ and $d_{30}$ for the composite sands, result in an increased flow resistance with respect to the reference sands at equal median grain size $\left(d_{50}\right)$ The $a$ coefficients increased by factors up to 1.68 and the $b$ coefficients increased by factors up to 1.44 with respect to the reference sands.

- The modified Ergun equation could provide accurate estimates of coefficients $a$ and $b$. The use of Ergun constants $A=183.8$ and $B=2.53$ and the use of $d_{30}$ as characteristic pore length are suggested to provide good fits. While using the method of Herrera and 
Felton (1991) with a characteristic pore length of $d_{m}-\sigma$, Ergun constants $A=139.1$ and $B=2.2$ are suggested.

- The derived Ergun constants of the current study are in agreement with the Ergun constants derived from other experimental datasets.

- We found a clear correlation between the experimentally derived Forchheimer coefficients $a$ and $b$ for our dataset of subangular-subrounded, uniformly graded filter sands at an optimal compaction grade.

Acknowledgements This work was supported by foundations STW (Foundation for Technical Sciences) and O2DIT (Foundation for Research and Development of Sustainable Infiltration Techniques). The authors thank Tony Valkering and Theo van Velzen from dewatering company Theo van Velzen for constructing the experimental setup and Peter de Vet from dewatering company P.J. de Vet \& Zonen for optimizing setup. We are grateful to Willem Jan Dirkx MSc for his help and constructive input during the nonlinear flow experiments Funding was provided by Stichting voor de Technische Wetenschappen (Grant No. 13263).

Open Access This article is distributed under the terms of the Creative Commons Attribution 4.0 International License (http://creativecommons.org/licenses/by/4.0/), which permits unrestricted use, distribution, and reproduction in any medium, provided you give appropriate credit to the original author(s) and the source, provide a link to the Creative Commons license, and indicate if changes were made.

\section{Appendix 1}

See Fig. 9.

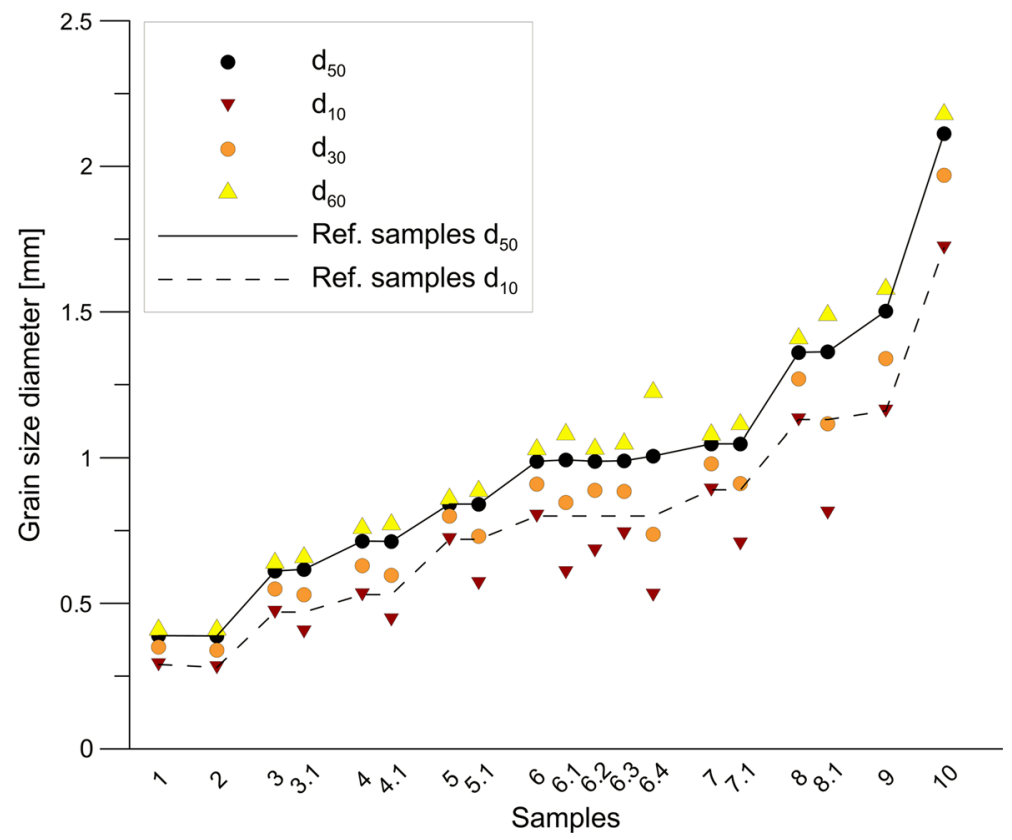

Fig. 9 The grain size distribution characterization $\left(d_{10}, d_{20}, d_{50}\right.$ and $\left.d_{60}\right)$ for the uniformly graded filter sands (S.1-10) and the sand mixtures (S3.1-8.1). Note that the coarse gravel (S.11 with $\left.d_{50}=6.34 \mathrm{~mm}\right)$ is not taken into account 


\section{Appendix 2}

See Fig. 10.
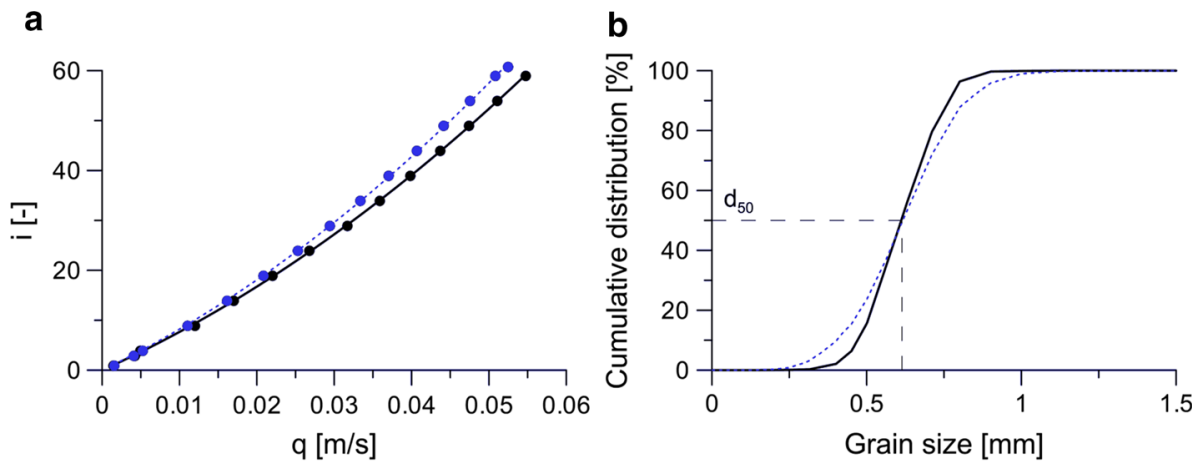

Sample 3 (ref.)

Sample 3.1
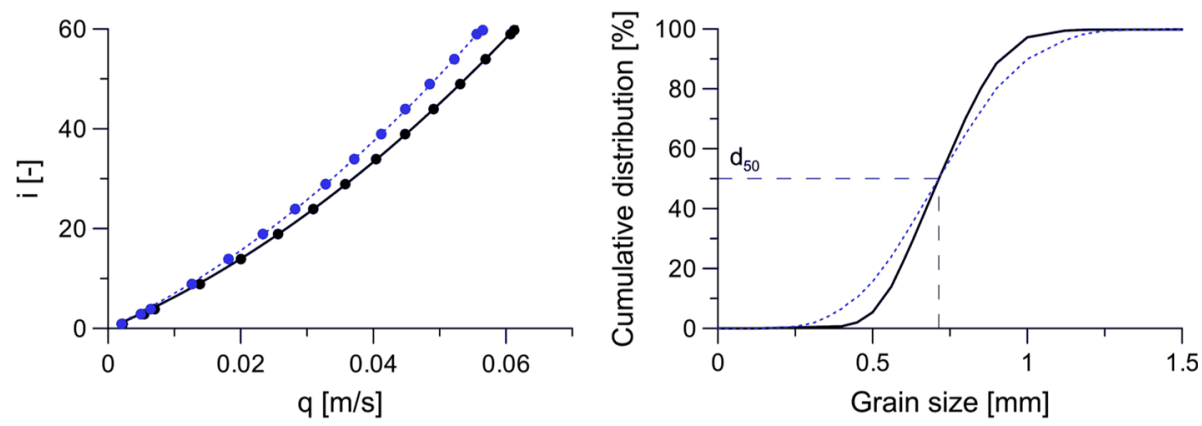

Sample 4 (ref.)

Sample 4.1
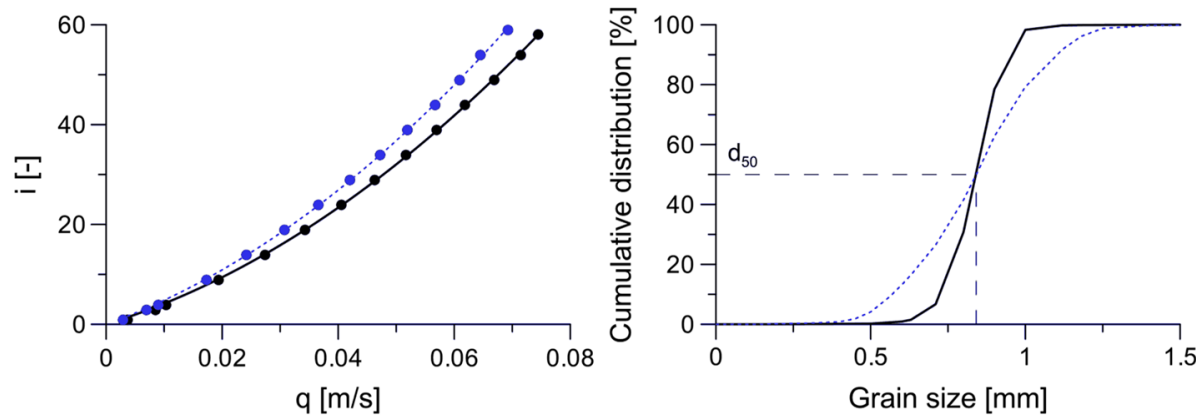

Sample 5 (ref.)

Sample 5.1

Fig. 10 a The $i-q$ plots for the sandy porous media S3.1-8.1 and $\mathbf{b}$ the cumulative grain size distributions 

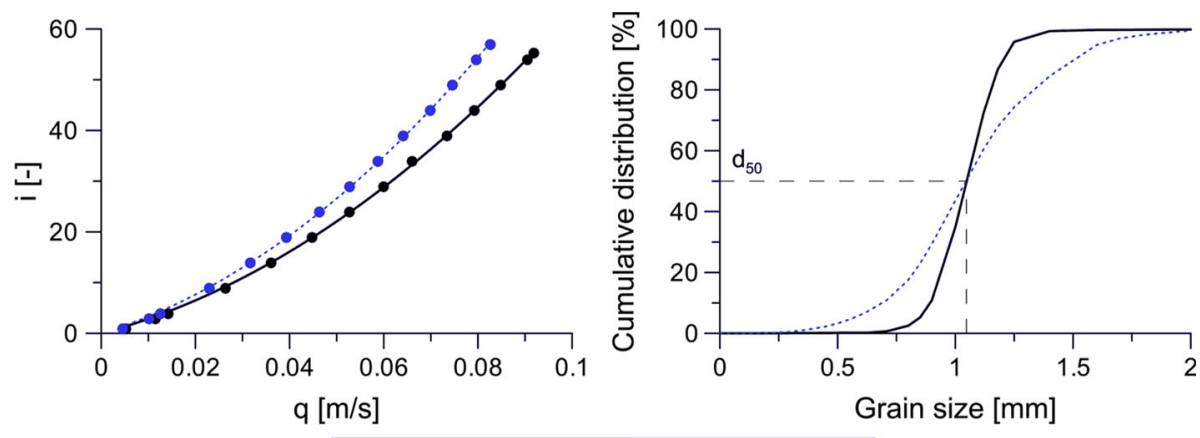

Sample 7 (ref.)

Sample 7.1
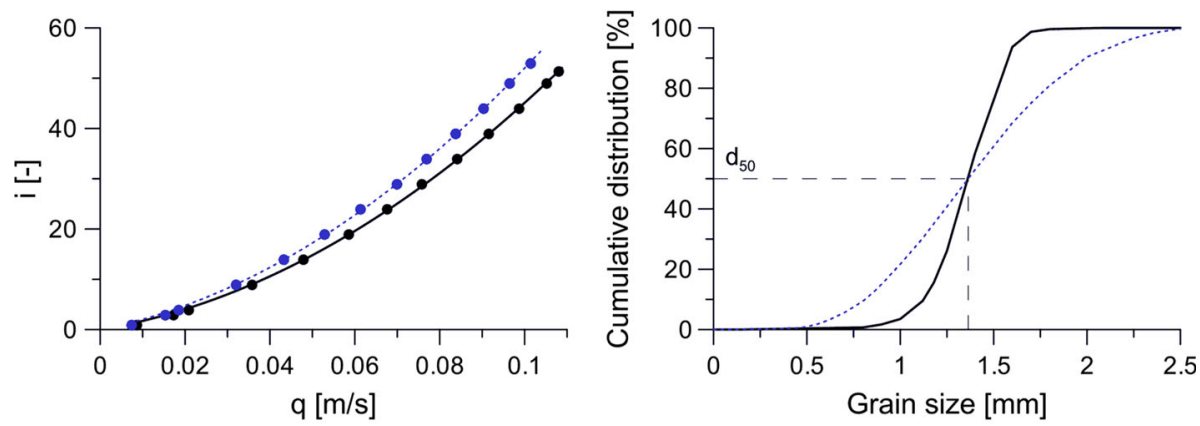

Sample 8 (ref.)

Sample 8.1

Fig. 10 continued

\section{References}

Ahmed, N., Sunada, D.K.: Nonlinear flow in porous media. J. Hydraul. Div. ASCE 95(6), 1847-1857 (1969)

Allen, K.G., von Bäckström, T.W., Kröger, D.G.: Packed bed pressure drop dependence on particle shape, size distribution, packing arrangement and roughness. Powder Technol. 246, 590-600 (2013)

Andersen, O.H., Burcharth, H.F.: On the one-dimensional steady and unsteady porous flow equation. Coast. Eng. 24, 233-257 (1995)

Arbhabhirama, A., Dinoy, A.A.: Friction factor and Reynolds number in porous media flow. J. Hydraul. Div. ASCE 99(6), 901-911 (1973)

Aulisa, E., Ibragimov, A., Valko, P., Walton, J.: Mathematical framework of the well productivity index for fast Forchheimer (non-Darcy) flows in porous media. Math. Models Methods Appl. Sci. 19(8), 1241-1275 (2009)

Bağci, Ö., Dukhan, N., Özdemir, M.: Flow regimes in packed beds of spheres from pre-Darcy to turbulent. Transp. Porous Med. 104(3), 501-520 (2014)

Bear, J.: Dynamics of Fluids in Porous Media. Dover Publications, New York (1972)

Blott, S.J., Pye, K.: GRADISTAT: a grain size distribution and statistics package for the analysis for unconsolidated sediments. Earth Surf. Process. Landf. 26(11), 1237-1248 (2001)

Bordier, C., Zimmer, D.: Drainage equations and non-Darcian modeling in coarse porous media or geosynthetic materials. J. Hydrol. 228(3-4), 174-187 (2000)

Carman, P.C.: Fluid flow through granular beds. Trans. Inst. Chem. Eng. 15, 150-166 (1937)

Chaudhary, K., Cardenas, M.B., Den, W., Bennett, P.C.: Pore geometry effects on intrapore viscous to inertial flows and on effective hydraulic parameters. Water Resour. Res. 49(2), 1149-1162 (2013) 
Chauveteau, G., Thirriot, C.: Régimes d'écoulement en milieu poreux et limite de la loi de Darcy [Regimes of flow in porous media and the limitations of the Darcy law]. La Houille Blanche 1(22), 1-8 (1967)

Comiti, J., Renaud, M.: A new model for determining mean structure parameters of fixed bed from pressure drop measurements: application to beds with packed parallelepipepal particles. Chem. Eng. Sci. 44(7), 1539-1545 (1989)

Comiti, J., Saribi, N.E., Montillet, A.: Experimental characterization of flow regimes in various porous media3: limit of Darcy's or creeping flow regime for Newtonian and purely viscous non-Newtonian fluids. Chem. Eng. Sci. 55(15), 3057-3061 (2000)

Darcy, H.: Les fontaines publiques de la ville de Dijon, p. 647. Victor Dalmont, Paris (1856)

Du Plessis, J.P.: Analytical quantification of coefficient in the Ergun equation for fluid friction in packed beds. Transp. Porous Med. 16(2), 189-207 (1994)

Dybbs, A., Edwards, R.V.: A new look at porous media fluid mechanics. Darcy to turbulent. Fundamentals of Transport Phenomena in Porous Media. Part of the NATO ASI Series book series (NSSE), Martinus Nijhoff, Dordrecht, vol. 82, pp. 199-256 (1984)

Ergun, S.: Fluid flow through packed columns. Chem. Eng. Prog. 48(2), 89-95 (1952)

Engelund, F.A.: On the laminar and turbulent flows of groundwater through homogenous sand. Danish Academy of Technical Science, Copenhagen (1953)

Fand, R.M., Kim, B.Y.K., Lam, A.C.C., Phan, R.T.: Resistance to the flow of fluids through simple and complex porous media whose matrices are composed of randomly packed spheres. J. Fluids Eng. 109(3), 268-273 (1987)

Fand, R.M., Thinakaran, R.: The influence of the wall on flow through pipes packed with spheres. J. Fluids Eng. 122(1), 84-88 (1990)

Firdaouss, M., Guermond, J.L., Le Quéré, P.: Nonlinear correction to Darcy's law at low Reynolds numbers. J. Fluid Mech. 343, 331-350 (1997)

Forchheimer, P.H.: Wasserbewegung durch boden. Z. Ver. Deutsch. Ing. 50, 1781-1788 (1901)

Fourar, M., Radilla, G., Lenormand, R., Moyne, C.: On the non-linear behavior of a laminar single-phase flow through two and three-dimensional porous media. Adv. Water Resour. 27(6), 669-677 (2004)

Geertsma, J.: Estimating the coefficient of inertial resistance in fluid flow through porous media. Soc. Petrol. Eng. J. 14(5), 445-450 (1974)

Hassanizadeh, S.M., Gray, W.G.: High velocity flow in porous media. Transp. Porous Med. 2(6), 521-531 (1987)

Herrera, N.H., Felton, G.K.: Hydraulics of flow through a rockfill dam yusing sediment-free water. Trans. ASABE 34(3), 871-875 (1991)

Hill, R.J., Koch, D.L.: The transition from steady to weakly turbulent flow in a close-packed ordered array of spheres. J. Fluid Mech. 465, 59-97 (2002)

Holditz, S.A., Morse, R.A.: The effects of non-Darcy flow on the behavior of hydraulically fractured gas wells. J. Pet. Technol. 28(10), 1179-1196 (1976)

Houben, G.J.: Review: Hydraulics of water wells flow laws and influence of geometry. Hydrogeol. J. 23(8), 1633-1657 (2015)

Huang, K., Wan, J.W., Chen, C.X., He, L.Q., Mei, W.B., Zhang, M.Y.: Experimental investigation on water flow in cubic arrays of spheres. J. Hydrol. 492, 61-68 (2013)

Izbash, S.V.: O filtracii V Kropnozernstom Materiale. USSR, Leningrad (1931). (in Russian)

Irmay, S.: On the theoretical derivation of Darcy and Forchheimer formulas. Trans. Am. Geophys. Union 39(4), 702-707 (1958)

Jafari, A., Zamankhan, P., Mousavi, S.M., Pietarinen, K.: Modeling and CFD simulation of flow behavior and dispersivity through randomly packed bed reactors. Chem. Eng. J. 144(3), 476-482 (2008)

Jolls, K.R., Hanratty, T.J.: Transition to turbulence for flow through a dumped bed of spheres. Chem. Eng. Sci. 21(12), 1185-1190 (1966)

Kadlec, H.R., Knight, L.R.: Treatment Wetlands. Lewis Publishers, Boca Raton (1996)

Kovács, G.: Seepage Hydraulics. Elsevier Scientific Publishing Company, Amsterdam (1981)

Koekemoer, A., Luckos, A.: Effect of material type and particle size distribution on pressure drop in packed beds of large particles: Extending the Ergun equation. Fuel 158, 232-238 (2015)

Lage, J.L.: The fundamental theory of flow through permeable media from Darcy to turbulence. In: Ingham, D.B., Pop, I. (eds.) Transport Phenomena in Porous Media, pp. 1-30. Pergamon, New York (1998)

Latifi, M.A., Midoux, N., Storck, A., Gence, J-N.: The use of micro-electrodes in the study of flow regimes in a packed bed reactor with single phase liquid flow. Chem. Eng. Sci. 44(11), 2501-2508 (1989)

Li, L., Ma, W.: Experimental study on the effective particle diameter of a packed bed with non-spherical particles. Transp. Porous Med. 89(1), 35-48 (2011)

Li, Z., Wan, J., Huang, K., Chan, W., He, Y.: Effects of particle diameter on flow characteristics in sand columns. Int. J. Heat Mass Transf. 104, 533-536 (2017) 
Liu, X., Civan, F., Evans, R.D.: Correlation of the non-Darcy flow coefficient. J. Can. Pet. Technol. 34(10), 50-54 (1995)

Macdonald, I.F., El-Sayed, M.S., Mow, K., Dullien, F.A.L.: Flow through porous media-the Ergun equation revisited. Ind. Eng. Chem. Fundam. 18(3), 199-208 (1979)

Mathias, S.A., Todman, L.C.: Step-drawdown tests and the Forchheimer equation. Water Resour. Res. 46(7), W07514 (2010)

Mathias, S.A., Moutsopoulos, K.N.: Approximate solutions for Forchheimer flow during water injection and water production in an unconfined aquifer. J. Hydrol. 538, 13-21 (2016)

Ma, H., Ruth, D.W.: The microscopic analysis of high Forchheimer number flow in porous media. Transp. Porous Med. 13(2), 139-160 (1993)

Mijic, A., Mathias, S.A., LaForce, T.C.: Multiple well systems with non-Darcy flow. Groundwater 51(4), 588-596 (2013)

Moutsopoulos, K.N., Tsihrintzis, V.A.: Approximate analytical solutions of the Forchheimer equation. J. Hydrol. 309, 93-103 (2005)

Moutsopoulos, K.N.: One-dimensional unsteady inertial flow in phreatic yaquifers, induced by a sudden change of the boundary head. Transp. Porous yMedia 70, 97-125 (2007)

Moutsopoulos, K.N., Papaspyros, I.N.E., Tsihrintzis, V.A.: Experimental investigation of inertial flow processes in porous media. J. Hydrol. 374(3-4), 242-254 (2009)

Nield, D.A.: Resolution of a paradox involving viscous dissipation and nonlinear drag in a porous medium. Transp. Porous Med. 41(3), 349-357 (2002)

Panfilov, M., Oltean, C., Panfilova, I., Buès, M.: Singular nature of nonlinear macroscale effects in high-rate flow through porous media. C. R. Mec. 331(1), 41-48 (2003)

Panfilov, M., Fourar, M.: Physical splitting of nonlinear effects in high-velocity stable flow through porous media. Adv. Water Resour. 29(1), 30-41 (2006)

Ranganadha Rao, R.P., Suresh, C.: Discussion of 'Non-linear flow in porous media', by N Ahmed and DK Sunada. J. Hydraul. Div. ASCE 96(8), 1732-1734 (1970)

Rietdijk, J., Schenkeveld F., Schaminée, P.E.L., Bezuijen A.: The drizzle method for sand sample preparation. In: Proceedings of the 6th International Conference on Physical Modelling, editor, Laue, Springman, Seward, pp. 267-272 (2010)

Rode, S., Midoux, N., Latifi, M.A., Storck, A., Saatdjian, E.: Hydrodynamics of liquid flows in packed beds: an experimental study using electrochemical shear rate sensors. Chem. Eng. Sci. 49(6), 889-900 (1994)

Salahi, M-B., Sedghi-Asl, M., Parvizi, M.: Nonlinear flow through a packed-column test. J. Hydrol. Eng. 20(9) (2015). doi:10.1061/(ASCE)HE.1943-5584.0001166

Schneebeli, G.: Expériences sur la limite de validité de la loi de Darcy et l'apparition de la turbulence dans un écoulement de filtration. La Houille Blanche 141, 141-149 (1955). (in French)

Scheidegger, A.E.: The Physics of Flow Through Porous Media, 3rd edn. University of Toronto Press, Toronto (1974)

Sedghi-Asl, M., Rahimi, H.: Adoption of Manning's equation to 1D non-yDarcy flow problems. J. Hydraul. Res. 49(6), 814-817 (2011)

Sedghi-Asl, M., Rahimi, H., Salehi, R.: Non-Darcy flow of water through a packed column test. Transp. Porous Med. 101(2), 215-227 (2014)

Seguin, D., Montillet, A., Comiti, J., Huet, F.: Experimental characterization of flow regimes in various porous media-2: Transition to turbulent regime. Chem. Eng. Sci. 53(22), 3897-3909 (1998)

Sen, Z.: Nonlinear flow towards wells. J. Hydraul. Eng. 15(2), 193-207 (1989)

Sidiropoulou, M.G., Moutsopoulos, K.N., Tsihrintzis, V.A.: Determination of Forchheimer equation coefficients a and b. Hydrol. Proc. 21(4), 534-554 (2007)

Skjetne, E., Auriault, J.L.: High velocity laminar and turbulent flow in porous media. Transp. Porous Med. 36(2), 131-147 (1999)

Stephenson, D.: Rockfill in hydraulic engineering. Elsevier, Amsterdam (1979)

Thauvin, F., Mohanty, K.K.: Network modeling of non-Darcy flow through porous media. Transp. Porous Med. 31(1), 19-37 (1998)

Tyagi, A.K., Todd, D.K.: Discussion of 'Non-linear flow in porous media', by N Ahmed and DK Sunada. J. Hydraul. Div. ASCE 96(8), 1734-1738 (1970)

Venkataraman, P., Rao, P.R.M.: Darcian, transitional, and turbulent flow through porous media. J. Hydraul. Eng. ASCE 124(8), 840-846 (1998)

Ward, J.C.: Turbulent flow in porous media. J. Hydraul. Div. ASCE 90(5), 1-12 (1964)

Wen, Z., Huang, G., Zhan, H.: Non-Darcian flow to a well in a leaky aquifer using the Forchheimer equation. Hydrogeol. J. 19(3), 563-572 (2011)

Wen, Z., Liu, K., Chen, X.: Approximate analytical solution for non-Darcian flow toward a partially penetrating well in a confining aquifer. J. Hydrol. 498, 124-131 (2013) 
Whitaker, S.: The Forchheimer equation: a theoretical development. Transp. Porous Med. 25(1), 27-61 (1996)

Yeh, H.D., Chang, Y.C.: Recent advances in modeling of well hydraulics. Adv. Water Resour. 51, 27-51 (2013)

Zhengwen, Z., Grigg, R.: A criterion for non-Dacry flow in porous media. Transp. Porous Med. 63(1), 57-69 (2006) 